\title{
Neurogenesis-on-Chip: Electric field modulated transdifferentiation of human mesenchymal stem cell and mouse muscle precursor cell coculture
}

\author{
Sharmistha Naskar ${ }^{\mathrm{a}, \mathrm{b}, \mathrm{d}, \mathrm{e}}$, Viswanathan Kumaran ${ }^{\mathrm{b}}$, Yogananda S. Markandeya ${ }^{\mathrm{c}}$, Bhupesh Mehta $^{\mathrm{c}}$, \\ Bikramjit Basu ${ }^{\text {a,d,e,* }}$ \\ ${ }^{a}$ Centre for Biosystems Science and Engineering, Indian Institute of Science, Bangalore, 560012, India \\ ${ }^{\mathrm{b}}$ Department of Chemical Engineering, Indian Institute of Science, Bangalore, 560012, India \\ ${ }^{c}$ Department of Biophysics, National Institute of Mental Health and Neurosciences, Bangalore, 560029, India \\ ${ }^{\mathrm{d}}$ Laboratory for Biomaterials, Materials Research Centre, Indian Institute of Science, Bangalore, 560012, India \\ ${ }^{\mathrm{e}}$ Centres of Excellence and Innovation in Biotechnology - Translational Centre on Biomaterials for Orthopaedic and Dental Applications, Materials Research Centre, IISc, \\ Bangalore, India
}

\section{A R T I C L E I N F O}

\section{Keywords:}

Lab-on-a-chip

Electric field

Human mesenchymal stem cell

Myoblast

Coculture

Neurogenesis

\begin{abstract}
A B S T R A C T
A number of bioengineering strategies, using biophysical stimulation, are being explored to guide the human mesenchymal stem cells (hMScs) into different lineages. In this context, we have limited understanding on the transdifferentiation of matured cells to another functional-cell type, when grown with stem cells, in a constrained cellular microenvironment under biophysical stimulation. While addressing such aspects, the present work reports the influence of the electric field (EF) stimulation on the phenotypic and functionality modulation of the coculture of murine myoblasts (C2C12) with hMScs [hMSc:C2C12 =1:10] in a custom designed polymethylmethacrylate (PMMA) based microfluidic device with in-built metal electrodes. The quantitative and qualitative analysis of the immunofluorescence study confirms that the cocultured cells in the conditioned medium with astrocytic feed, exhibit differentiation towards neural-committed cells under biophysical stimulation in the range of the endogenous physiological electric field strength $(8 \pm 0.06 \mathrm{mV} / \mathrm{mm})$. The control experiments using similar culture protocols revealed that while C2C12 monoculture exhibited myotube-like fused structures, the hMScs exhibited the neurosphere-like clusters with SOX2, nestin, BIII-tubulin expression. The electrophysiological study indicates the significant role of intercellular calcium signalling among the differentiated cells towards transdifferentiation. Furthermore, the depolarization induced calcium influx strongly supports neural-like behaviour for the electric field stimulated cells in coculture. The intriguing results are explained in terms of the paracrine signalling among the transdifferentiated cells in the electric field stimulated cellular microenvironment. In summary, the present study establishes the potential for neurogenesis on-chip for the coculture of hMSc and C2C12 cells under tailored electric field stimulation, in vitro.
\end{abstract}

\section{Introduction}

In the expanse of regenerative medicine, the adult mesenchymal stem cell is of great importance due to its potential to transdifferentiate into both mesenchymal and non-mesenchymal cell types under the influence of biophysical cues [1]. While reprogramming the stemness, a number of studies have reported the osteogenesis and chondrogenesis, in vitro [2]. Many researchers have further investigated mesenchymal stromal cell capacity to differentiate into multiple cell types of mesodermal and non-mesodermal origin, including endothelial cells [3], cardiomyocytes [4], hepatocytes [5] and neural cells [6,7].

The targeted manipulation of stem cells is considered a promising approach in the field of therapeutic tissue regeneration. The elucidation of the governing molecular pathways has made the process of transdifferentiation possible by exploiting the plasticity of adult stromal cells to generate various cellular lineages. The conversion of hMScs into neural cells is an excellent example of transdifferentiation and reprogramming of the cellular genetics. Several studies have also reported the genetic engineering approaches as well as microenvironmental influences on transdifferentiation [8-10].

The endogenous electric field is one of the biophysical cues to regulate the cellular behaviour. Yamada et al. reported that mild electrical stimulation strongly influences embryonic stem cells to assume neuronal fate [11]. Han et al. suggested that the surface charge

\footnotetext{
* Corresponding author. Laboratory for Biomaterials, Materials Research Centre, Indian Institute of Science, Bangalore, 560012, India.

E-mail address: bikram@iisc.ac.in (B. Basu).
} 
influences the cell more than the wettability and roughness of the substrate [12]. As an extension of their observation, the researchers have acknowledged that the differentiation of stem cells under the influence of external cues can open up possibilities for studying novel mechanisms underlying cellular differentiation and more importantly, suggested the possibilities for adaption in clinical contexts.

The proliferative potential and the propensity to differentiate towards multiple lineages makes the myoblast cell line suitable for generative engineering [13]. The myoblasts could be differentiated into osteocytes or adipocytes when cultured with bone morphogenetic proteins (BMPs) or adipogenic inducers, respectively. Such prior studies suggest myoblasts are a multipotent cell type with the capability to modulate its genetic decision to a non-muscular lineage [14-16]. Further, the induction of BMP into muscular tissues induces ectopic bone formation at the site of implantation, in vivo [16].

The present study is based on the hypothesis that both the stromal origin cells of $\mathrm{C} 2 \mathrm{C} 12$ and hMSc exhibit some degree of plasticity to support the genetic reprogramming $[17,18]$. The hypothetical pathway, leading to neural-like cells from the committed adult cells (C2C12), can be considered as dedifferentiation to achieve stemness and transdifferentiation to generate non-muscular cell type. To validate this hypothesis, we have fabricated lab-on-a-chip (LOC) with embedded metal electrodes, which facilitates the application of manipulative biophysical cues. The most important aspect of deploying microfluidic technology is to create a controlled cellular microenvironment, which can closely mimic the physiological conditions. Lindstrom et al. demonstarted that stem cell based bioengineering approaches could be studied using a combination of the robust $\mathrm{TiO}_{2}$ coating of the microwell chip, as this customised design enables thousands of individually separated single, or clones, of stem cells to be studied simultaneously [19]. We have demonstrated that hMScs can not only undergo self-differentiation, but also trigger other precursor cells to undergo transdifferentiation. The present work is based on the capability of the biophysical cues on the paracrine activity of the stem cells to alter the behaviour of the cocultured committed cells. It is worthwhile to mention that extra cellular matrix (ECM) provides a biochemical influence along with the biophysical cues towards tissue engineering, and has been proven to have angiogenic regenerative properties [20]. Hosseinkhani et al. have reported that the 3D culture using synthetic peptide (IKVAV) based collagen functionalised matrix provides physiological mimicry to the dorsal root ganglion (DRG) cells, and influence them to proliferate towards forming neural network [21]. The glycosylated collagen substrates were found to have the efficacy to induce differentiation of the human embryonic stem cells (hESC) into human embryonic stem cellderived hepatocyte-like cells (hESC-HLCs) [22]. Jahani et al. demonstrated that oxygen plasma treated scaffolds of poly-caprolactone differentiate the mesenchymal stem cells in the neuronal direction. The orientation of the ECM-like extracellular fibres are efficient to compel the cells towards neuronal differentiation [23]. 3D culture with composites of poly (glycolic acid) and collagen have been found to be very efficient for differentiation of mesenchymal stem cell (MSC), when incorporated with bone morphogenic protein 2 (BMP2) plasmid DNA, functionalised with dextran-spermine [24].

In the above context, the objective of the present study is to closely monitor the cocultured transdifferentiation of mixed cell population of hMSc and C2C12 under the influence of the electric field within a custom designed biomicrofluidic device. For this purpose, the hMSc cell line was cocultured along with myoblasts to establish the role of cell-tocell communication through paracrine signalling via co-operative differentiation, when the culture system was stimulated by electric field at the same time. The epigenetics of the transdifferentiation of the myoblasts will be investigated by qualitative/quantitative assays, including WST-1, flow cytometry, PCR, immunofluorescence assay, and electrophysiological analysis.

\section{Materials and methods}

\subsection{Fabrication of PMMA microfluidic devices with in-built electrodes}

The development of microfluidic culture system involves the design of lab-on-a-chip (LOC) layout, selection of materials, fabrication process, and sterilization technique. In the current work, the microfluidic channel layout was designed using the CorelDRAW X6 software. Polymethylmethacrylate (PMMA) was selected as the material for the device fabrication, as it is non-cytotoxic, biocompatible and autoclavable. The LOC devices with implanted electrodes were fabricated using three polymethyl methacrylate (PMMA, Plexiglas ${ }^{\circledR}$ USA) sheets of thickness $1 \mathrm{~mm}$ (Fig. 1a). The bottom-most layer of the device was used as a substrate for the cells. The middle layer of the PMMA sheet contained parallel through-cut channels of $2 \mathrm{~mm}$-width, $35 \mathrm{~mm}$-length and $1 \mathrm{~mm}$-height, made using a $\mathrm{CO}_{2}$ laser-cutting machine (Universal LASER, USA). The channel height is kept as $1 \mathrm{~mm}$, in commensurate with the thickness of the PMMA sheet. The top layer of PMMA sheet is used for sealing the channels from external environment, and it includes the inlet and outlet channels (both $1 \mathrm{~mm}$ in diameter), which are flush mounted with microtips to avoid leakage. The PMMA sheets are bonded together using two $3 \mathrm{M}^{\mathrm{TM}}$ pressure sensitive adhesive (PSA) films of $50 \mu \mathrm{m}$. It is important to mention that conducting PSA tape was used for bonding the top layer with middle layers and to enhance the electric field stimulation of the cells. To avoid misalignment of the patterns, these layers were stacked using the alignment screws.

Two parallel inbuilt electrodes (at a distance of $1 \mathrm{~mm}$ from the edge of the central chnanel) were placed on both sides of a central cellloading channel (which is width of $2 \mathrm{~mm}$ and length of $35 \mathrm{~mm}$ ). These electrodes were built with Field's metal, $\left(\mathrm{T}_{\mathrm{m}} \sim 62{ }^{\circ} \mathrm{C}\right)$, which is a eutectic alloy with nominal composition $51 \mathrm{wt} \% \mathrm{In}-32.5 \mathrm{wt} \% \mathrm{Bi}-16.5 \mathrm{wt}$ $\%$ Sn. Molten metal $\left(\mathrm{T} \sim 70^{\circ} \mathrm{C}\right)$ was filled into the electrode-channels of the LOC. The metal was then cooled, resulting in channels with conducting metal, serving as electrodes on the both sides of the central channel. These electrodes were connected to a direct current source (Digilog DC Source) (Fig. 1b). The advantages of using the in-built electrodes are four fold. First, the in-built electrodes provide good reproducibility as they were permanently fixed in the LOC. Secondly, the designed biomicrofluidic device can facilitate a better quantification of the electric fields, because of identical electrode dimension. Thirdly, the entire cell population could simultaneously be stimulated with uniform electric field, unlike the conventional petridish culture. The fourth advantage is the microfluidic design, which can be implemented to have a close resemblance to physiological endogenous electric field [25].

\subsection{Electric field application}

In this subsection, the methodology of electric field stimulation of cells within microfluidic device is briefly discussed. While mimicking the endogenous low continuous electric field, we have designed the experimental protocol to apply an electric field of strength $\sim 8 \pm 0.06 \mathrm{mV} / \mathrm{mm}$, continuously for 9 days at an exposure of $20 \mathrm{~h}$ / day, with a short intermittent resting phase of $4 \mathrm{~h}$ for each $24 \mathrm{~h}$ of the culture period. In all our experiments, the cell were initially grown for 3 days, before they experienced electrical stimulation (Fig. 1b). The electric field strength has been measured by recreating exact simulation of the experimental conditions. In such scenario, a microfluidic device has been filled with cell culture media, while the two parallel electrodes were connected to the DC source at $10 \mathrm{~V}$. In addition, two silver electrodes were placed just at the edges of the central channel, at different locations along the length (Fig. 1c and d). Two electrodes were connected to a high precision multimeter (resolution up to the $4^{\text {th }}$ decimal place). The electric field strength (E) at these locations across the channel was then determined by the ratio of potential difference $(\mathrm{V})$ to the distance (D) between the electrodes. In Fig. 1d, the plot of $\mathrm{E}$ as a 


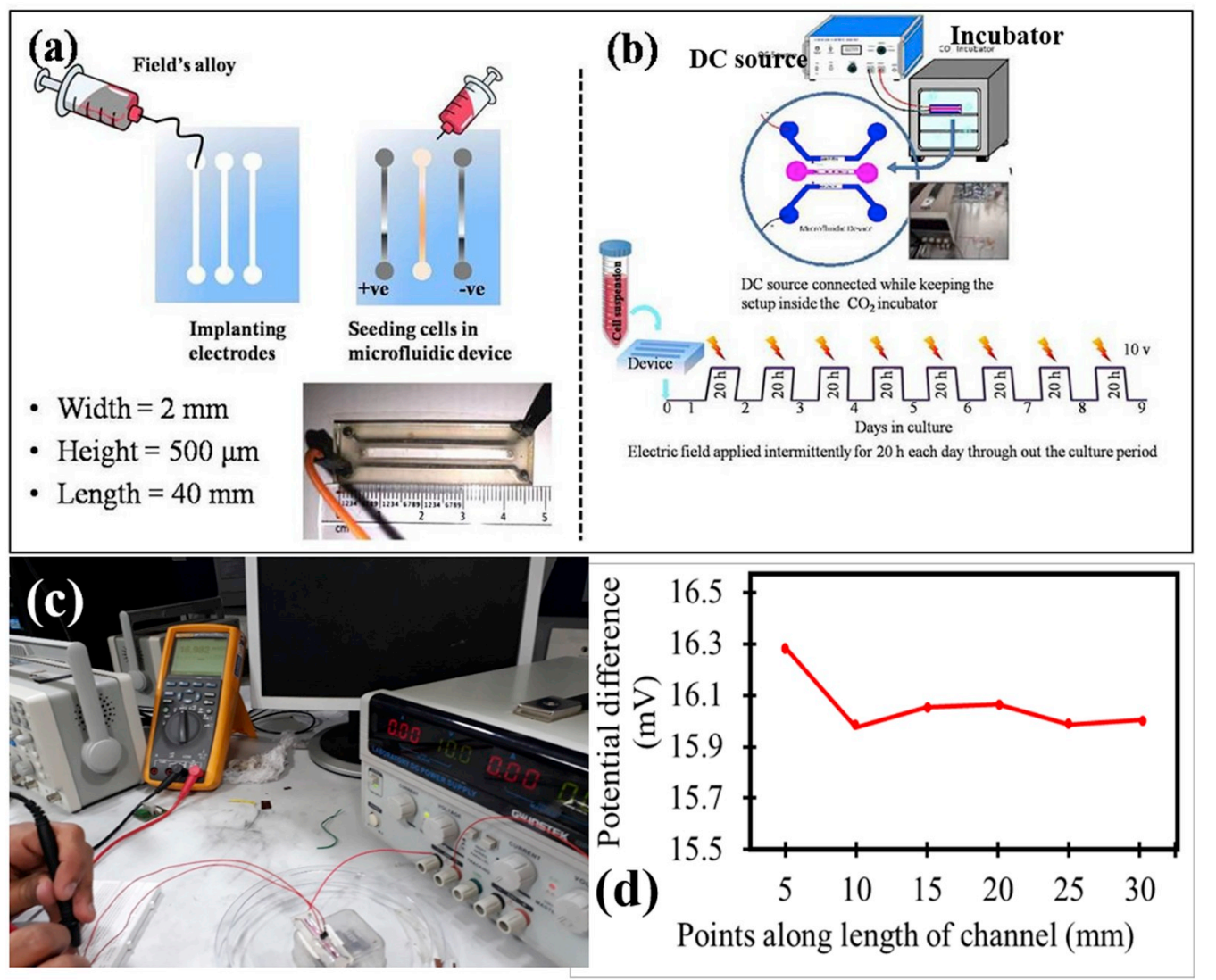

Fig. 1. Biomicrofluidics has enabled the study of EF-mediated transdifferentiation of C2C12, cocultured with hMSc in physiologically simulated culture condition. (a) Field's alloy is used to build the electrodes of the three channeled microfluidic device. (b) Cell culture setup using DC source to define electric field stimulation to culture. (c) Instrumental measurement of the electric potential across the width of the PMMA-based cell culture-channel with application of $10 \mathrm{~V}$ to the device-electrodes. (d) Electric potential across the channel, measured at different points along the length of the channels of width $2 \mathrm{~mm}$. function of distance along the channel is provided. The electric field varied within the range of $8 \pm 0.06 \mathrm{mV} / \mathrm{mm}$.

\subsection{Cell culture experiments in LOC device}

The non-hematopoietic stem/progenitor cell source of human bone marrow is commonly called as human mesenchymal stem cells or hMSc. As the name suggests, hMScs are produced from the mesenchymal tissue of the developing fetus. It is isolated from Wharton's jelly and umbilical cord blood as an embryonic source as well as available from molar tooth bud and bone marrow, as an adult source. The hMScs used in this study were characterised by expression of positive and negative markers (Fig. S1). These multipotent stromal cells, can be differentiated into both mesenchymal and non-mesenchymal origin cells, including neural lineage [26] with stem cell behaviour being regulated by biophysical cues $[1,27]$.

The $\mathrm{C} 2 \mathrm{C} 12$ cell line was the mouse pluripotent mesenchymal precursor, obtained from the American Type Culture Collection (ATCC). The rationale behind choosing the $\mathrm{C} 2 \mathrm{C} 12$ cell line includes the fact, that (a) they are the multipotent cells with the ability to undergo differentiation to adipocytes, osteocytes, neural lineage in the presence of suitable cues and, that (b) they have good adherence onto the surfaces without compromising the reproducibility of the experimental outcomes.

Both of these cells (hMSc and C2C12) were cocultured in the ratio of 1:10 under the electric field stimulation in the custom designed LOC. The cellular morphology of the coculture could be observed in Fig. S2, as the tagged $\mathrm{C} 2 \mathrm{C} 12$ cells have successfully expressed the RFP-tubulin along with untagged hMSc. C2C12 cell tagging was performed with CellLight $^{\mathrm{TM}}$ Tubulin-RFP, BacMam 2.0, following manufacturer's protocol. The transdifferentiating potential of myoblasts, together with stem cells, were performed with the prior approval of the Institutional Committee for Stem Cell Research and Therapy (IC-SCRT), Indian Institute of Science, Bangalore.
Three sets of experiments were carried out to understand the cellular behaviour of the cocultured cells under the influence of electric field. They were a) coculture of hMSc and C2C12 under electric field, b) monoculture of hMSc under electric field, c) monoculture of $\mathrm{C} 2 \mathrm{C} 12$ under electric field. For every set, the control was considered as the cells growing without the electric field. The days were counted from the day of the applying the electric field. The protocol for electric field application was optimized through several trial attempts and finalized as $20 \mathrm{~h}$ a day for 9 days in culture, as mentioned in Subsection 2.2. As control experiements, monoculture of $\mathrm{C} 2 \mathrm{C} 12$ and hMSc were also exposed to electric field stimulation in parallel, keeping the culture-protocol identical with experimental group. Before the cells were exposed to electric field, they were allowed to grow for 3 days to allow them to physically adapt inside the device.

The cells were cultured using two different media; (i) proliferation media (before applying electric field for 3 days) and (ii) differentiation media (during application of electric field from day 4 for next 9 days). The control groups were not exposed to electric field, but they were also grown in similar media in parallel to the experimental groups with identical culture timeline. It is worthwhile to mention that the cell culture media plays a supportive role along with the external stimulus to facilitate the differentiation process. The researchers usually prefer to use the conditioned media on top of the external stimuli to differentiate the cells $[28,29]$.

Proliferation media: Complete culture medium containing DMEM (Dulbecco's Modified Eagle's Medium; Invitrogen), supplemented with 20\% Mesenchymal Stem Cell - Fetal bovine serum (MSC-FBS; Invitrogen), $1 \%$ antibiotic-antimycotic solution (Sigma) was used for the first three days in culture, to allow the cells to adapt in biophysical environment in LOC before applying the electric field. The cells were maintained at $37^{\circ} \mathrm{C}$ in a saturated humidified atmosphere, containing $5 \% \mathrm{CO}_{2}$. Prior to the seeding, the cells were revived in a tissue culture graded T25 flask (Eppendorf, Germany). Upon reaching 70\%-80\% confluency, the cells were used for the experiment. They were 
harvested using $0.05 \%$ Trypsin-EDTA (Invitrogen) and sub-cultured for further use. The cells were centrifuged at $900 \mathrm{rpm}$ for $4 \mathrm{~min}$ and then re-suspended in the complete growth medium in order to achieve single cell suspension of cell concentration of $\sim 10^{4}$ cells per ml of media for further use.

Differentiation media: A specialized media was prepared for the differentiation experiements with the neurobasal media (Invitrogen), $200 \mu \mathrm{M}$ ascorbate and $2 \mathrm{mM}$ glutamine (Invitrogen) along with $2 \% \mathrm{~B}-27$ supplement. $90 \%$ of this media was mixed with $10 \%$ of primary astrocytic feed (media collected from astrocytes cultured of 5 days). The astrocytic feed was included to enhance the neural microenvironment.

The single-cell suspension of the hMScs, was used first for seeding into the microfluidic LOC devices for the coculture experiment. After a gap of $24 \mathrm{~h}, \mathrm{C} 2 \mathrm{C} 12$ cells were seeded inside the culturing channel. It is worth mentioning that before the cells were seeded, the channel was coated with rat tail type-I collagen for better adherence of the cells.

\subsection{Cellular viability and functionality analysis}

\subsubsection{Cell viability using WST-1 assay}

The WST-1 assay was chosen for evaluating cell viability. This specific biochemical assay was performed using water-soluble tetrazolium salts (WST-1, 4-[3-(4-iodophenyl)-2-(4-nitrophenyl)-2H-5-tetrazolio]-1,3-benzene-disulfonate, Roche) [30]. The WST-1 assay was performed after the prescribed culture durations of 3 days, 5 days and 9 days. To perform the colorimetric WST-1 (Roche) assay, 10\% v/v solution of WST-1 salt and complete culture medium was prepared and was injected into each of the devices. The samples were incubated for $4 \mathrm{~h}$ at $37^{\circ} \mathrm{C}$ in $5 \% \mathrm{CO}_{2}$ with $95 \%$ humidity.

As part of the microfluidic culture, the pooling of the cell samples (maintained under identical conditions) was performed as the fluid volume from a single channel was less for spectroscopic detection. We collected $200 \mu \mathrm{L}$ of the fluid and the optical density (OD) of the developed color was measured by a microplate reader (iMark, Biorad laboratories) at a wavelength of $450 \mathrm{~nm}$. The absorbance value provides a direct correlation to the number of proliferatingcells for each sample. The measurements are reported after averaging the data from three wells repeated thrice. Cell viability (or proliferation) was calculated using the following equation;

$$
\begin{aligned}
& \% \text { cell viability } \\
& =\frac{\text { Mean absorbance of the sample }- \text { Absorbance of the blank }}{\text { Mean absorbance of the control }- \text { Absorbance of the blank }} \times 100
\end{aligned}
$$

\subsubsection{FDA/PI staining for live/dead cells}

For assessing live and dead cells, the cells grown in LOC device were stained with fluorescein diacetate (FDA) and propidium iodide (PI), respectively. It is a double staining method for the live samples. The staining was performed with $1 \mathrm{~mL}$ of $25 \mu \mathrm{g} / \mathrm{mL}$ of FDA for $15 \mathrm{~min}$, maintained at $37^{\circ} \mathrm{C}$. Subsequently, the cell samples were stained with the counter red stain with $1 \mathrm{~mL}$ of $10 \mu \mathrm{g} / \mathrm{mL}$ of PI for $5 \mathrm{~min}$ at room temperature. After staining, the samples were washed thrice with $1 \times$ PBS to remove excess stain and imaged under Nikon fluorescence microscope with FITC and Texas Red filter (Nikon LV 100D, Japan).

\subsubsection{Immunostaining of actin filaments and microtubules}

The cell samples were washed twice with $1 \times$ PBS and then added to $4 \%$ paraformaldehyde solution (PFA, SD Fine-Chem Lit), before it was held for $30 \mathrm{~min}$. Once the cells are fixed, the covering PMMA sheets were carefully removed and the bottom most sheet, was retained for staining. This minimizes the difficulty of pumping reagents into the channel. The samples were washed three times with $1 \times$ PBS and $0.1 \%$ Triton $\mathrm{X}$ was used for permeation of the samples. The cell samples were blocked with $1 \%$ bovine serum albumin (BSA) for $1 \mathrm{~h}$ to prevent unwanted binding of the dye, leading to false positive results as well as to reduce background signal due to non-specific binding. Prior to microscopic observations, actin and microtubules were stained with an anti- $\alpha$-tubulin antibody, labelled with phalloidin-rhodamine (Invitrogen) and Alexa Fluor 488 (Invitrogen), respectively for $1 \mathrm{~h}$. DAPI (Invitrogen) was used to stain the nuclear chromatin. Any excess stain was removed by washing with $1 \times$ PBS and the cells were observed under a fluorescence microscope (Nikon LV 100D, Japan).

\subsubsection{Primary staining for selected neural markers}

Multi-labelling of combinatorial marker expression establishes the reliability of the results of a canonical stem cell research. In the present study, a combination of specific markers was used to avoid erroneous inferences. For example, nestin is a neural marker, which is perhaps best known to characterize the neuronal precursor cells. The immature or incompletely differentiated cells express the nestin, which is decreased later as the neural cell matures in the lineage [31]. Any study on neurogenesis widely focuses on the class III beta-tubulin protein ( $\beta$ III-tubulin), as it is being selectively expressed in neuronal cells during the progression of the neurogenesis [32]. The MAP2 proteins serve to stabilize the architecture of the neurites by microtubular interaction and hence, growth by establishing crosslinking with the intermediate filaments. These neuron-specific cytoskeletal associated proteins are found to be more intensely distributed in the dendrites along with the axon, implicating its role in deciding neural protrusion and giving the functional shape during neuron development [33].

The presence of the above-mentioned marker proteins was evaluated by immunocytochemistry at the end of day 9. For this purpose, the cells were fixed with $4 \%$ paraformaldehyde (PFA) for $30 \mathrm{~min}$. For immunostaining, the cells were permeabilized with $0.1 \%$ Triton-X in PBS for $5 \mathrm{~min}$ and 1\% BSA was used for $30 \mathrm{~min}$ to block non-specific staining of antibodies. The primary antibody labelling with nonfluorescent antibody was carried out by incubating in a 1:200 dilution of antibody solutionat room temperature for $1 \mathrm{~h}$. The working solution of antibody was prepared using dilution buffer $(1 \%$ BSA $+0.01 \%$ Triton- $X$ in PBS). After subsequent washing, secondary antibody, labelled with FITC, was added at a dilution of 1:500 (anti-mouse FITCconjugate). Before adding the secondary antibody, the washing of the cell samples with $1 \times$ PBS is crucial in order to avoid false positive results. Complementary staining of the actin filaments and nucleus were performed using rhodamine-phalloidin conjugate (Invitrogen) and DAPI (Invitrogen). The imaging was carried out using fluorescence microscope (Nikon LV 100D) with appropriate filters of specified wavelengths with oil immersion lenses.

\subsubsection{Quantitative gene expression study by $q R T-P C R$}

Quantitative-reverse-transcription PCR (qRT-PCR) was carried out using a one-step cell-to-CT, qRT-PCR kit procured from Invitrogen (A24571, Ambion). This specific PCR kit was chosen considering the challenge of extracting mRNA from a very low number of cells, even after sample pooling. The protocol was set according to the manufacturer's instructions with thoughtful, but minor modification to minimize the procedural loss of mRNA. As the channels could accommodate a low number of cells, the cell lysis solution was added directly to the adhered cells, instead of enzymatic lifting. Primer solution at strength of $200 \mathrm{nM}$ was added to $20 \mu \mathrm{L}$ of reaction volume, which consists of $10 \mu \mathrm{L}$ of the qRT-PCR mix and $0.16 \mu \mathrm{L}$ of the RT-PCR mix. The volume was made up to the above-mentioned desired amount by adding nuclease-free water. The specifications for running the PCR reaction, include 40 cycles at $95^{\circ} \mathrm{C}$ for $30 \mathrm{~s}$, followed by $70^{\circ} \mathrm{C}$ for $1 \mathrm{~min}$, and lastly at $72^{\circ} \mathrm{C}$ for $1 \mathrm{~min}$. All the PCR cycles started in the hotstart mode at $95^{\circ} \mathrm{C}$ for $3 \mathrm{~min}$ and terminated at $72{ }^{\circ} \mathrm{C}$ for $2 \mathrm{~min}$.

It is worth mentioning that for each gene, three sets were prepared to validate the outcome. GAPDH was considered as the housekeeping gene, against which the gene expression is considered to be upregulated or downregulated. The gene expression was evaluated in the form of 
Table 1

Primers used for qRT-PCR.

\begin{tabular}{|c|c|c|}
\hline Target gene & Forward & Reverse \\
\hline nes (Nestin) & 5'GGGAAGAGGTGATGGAACCA-3' & 5’AAGCCCTGAACCCTTTGC-3' \\
\hline map2 (MAP2) & 5'TTGGTGCCGAGTGAGAAGA-3' & 5’GTCTGGCAGTGGTTGGTTAA-3' \\
\hline Tubb3 (ßIII-Tubulin) & 5’GCGGATCAGCGTCTACTACA-3' & 5'CACATCCAGGACCGAATCCA-3' \\
\hline GAPDH & 5'-GACCCCTTCATTCATTGACCTCAACTACAT-3' & 5'-TGATGGCATGGACTGTGGTCATGA-3' \\
\hline
\end{tabular}

$2^{-\Delta \Delta \mathrm{CT}}$ (CT, cycle threshold) values. ROX was the inbuilt reference dye to rule out the plate-to-plate variation of fluorescence. PCR primers were designed using NCBI based primer designing tools. The parameters, like Guanine-cytosine (GC) content, length, and exclusion of complementary sequence were strictly maintained, while designing the primers to get an optimum output (Table 1).

\subsubsection{Fluorescence assisted cell sorter (FACS) analysis}

To determine the cell fate processes quantitatively, FACS analysis for BIII-tubulin expression was conducted. The cells were enzymatically lifted from the channels and pooled as per groups (C2C12 monoculture with and without electric field, hMSc monoculture with and without electric field, hMSc/C2C12 coculture with and without electric field) to attain $10^{4} \mathrm{cell} / \mathrm{ml}$. $1 \mathrm{ml}$ of cell suspension was added to $500 \mu \mathrm{l}$ of $4 \%$ paraformaldehyde for $1 \mathrm{~h}$, while keeping the samples at $4^{\circ} \mathrm{C}$. The paraformaldehyde-cell mixture was pelleted by centrifugation and the pellet was added to $0.1 \%$ triton- 100 . Similarly, the pellet was recovered and dispersed into equal amount $(50 \mu \mathrm{l})$ of nestin primary antibody (1:200). This was kept for $4 \mathrm{~h}$. The pellet was washed and added with secondary alexafluor tagged antibody $(50 \mu \mathrm{l}$ of $1: 1000)$ and DAPI $(50 \mu \mathrm{l})$. After waiting for $1 \mathrm{~h}$, the cell samples were washed and restored in $1 \mathrm{ml}$ of PBS. The readings were assessed with flow cytometry runs for minimum of 1000 events with low speed flow. The recorded data were analyzed using BD FACS-Express.

\subsubsection{Functional analysis of cells by fluorescence imaging and patch clamp technique}

To assess the physiological property of cells in culture, $\mathrm{KCl}$ depolarization induced change in intracellular calcium by fluorescence imaging, and along with this, whole-cell patch-clamp recordings were performed. Cultured cells, adhered to Type 1 collagen coated coverslips, were bath perfused with HEPES buffer of composition (in $\mathrm{mM}$ ): $130 \mathrm{NaCl}, 1.8 \mathrm{CaCl}_{2}, 5 \mathrm{KCl}, 20$ HEPES, 6 glucose. The $\mathrm{pH}$ was adjusted to 7.4 with $\mathrm{NaOH}$ and osmolarity to $300-310$ mOsm.

2.4.7.1. Intracellular calcium imaging. The cultured cells, plated over poly-L-lysine coated cover slip were incubated for $45 \mathrm{~min}$ at $37^{\circ} \mathrm{C}$ with $\mathrm{Ca}^{2+}$ sensitive fluorescent dye, Fluo- $4 \mathrm{AM}(5 \mu \mathrm{M})$ with pluronic acid $(0.01 \%)$ in HEPES buffer [34,35]. Cells were subsequently washed with fresh HEPES and kept in dark for $15 \mathrm{~min}$ for intracellular deesterification of Fluo-4 AM. The fluorescent dye loaded cells were further imaged under the fluorescent microscope (Olympus BX61, Japan). The bath volume of the chamber was $10 \mathrm{ml}$ and the flow rate for perfusion of buffer was $1.5 \mathrm{ml}$ per minute. During microscopic observations, the cells were excited at $495 \mathrm{~nm}$ and emission captured at $515 \mathrm{~nm}$. The images were acquired using RETIGA A 2000DC camera. The change in $\left[\mathrm{Ca}^{2+}\right]_{i}$ in single neurons was represented as change in Fluo-4 fluorescence intensity, as measured using by Image $\mathrm{J}$ software (NIH, USA).

2.4.7.2. Whole-cell patch-clamp recordings. Whole cell patch clamp recording was carried out using the HEKA EPC10 amplifier (HEKA, Germany). Prior to such measurements, patch pipettes (5-10 M $\Omega$ ) were fabricated from borosilicate glass capillary (OD $1.5 \mathrm{~mm}$; World Precision Instrument, WA, USA) using a P-2000 micropipette puller (Sutter Instrument, Novato, CA, USA). The pipettes were filled with internal buffer of composition (in $\mathrm{mM}$ ): $130 \mathrm{~K}$-gluconate, $4 \mathrm{Mg}$-ATP, 11 EGTA, 10 HEPES-KOH, and $1 \mathrm{CaCl}_{2}$. The buffer solution was characterised by $\mathrm{pH} \quad 7.2$ and Osmolarity 290-295 mOsm. Additionally, $2 \mathrm{mM}$ QX314 was added to the internal solution to block sodium current. The holding potential of the cells was kept at $-60 \mathrm{mV}$. During the measurements, access resistance was $<20 \mathrm{M} \Omega$ and the recorded currents were low-pass filtered at $2-4 \mathrm{kHz}$. The analysis was performed in Igor Pro and plotted in Origin 2016 software.

\subsection{Statistical analysis}

All the results were expressed as mean \pm standard error (SE). Student's t-test and one-way ANOVA with post-hoc Tukey's test were performed to reveal the statistical differences among the different samples (electric field exposed and control, without the electric field stimulation). All the statistical analysis was performed using SPSS-16.0 software (SPSS Inc.@2010). The cut-off p-values were set at 0.05 and 0.01 , lower than which were considered to be too low to accept the null hypothesis.

\section{Results}

Simao et al. reported the capability of perfusion based bioreactor to recapitulate the brain-like environment, which can facilitate neuronal differentiation of the iPSc-derived neural stem cells [36]. Such microenvironment plays critical role in cellular decision. Burdick et al. justified the need of the microenvironment adjustment to unlock the full pledged potential of the stem cells [37]. In order to create biophysically simulated microenvironment for the cellular differentiation, the present study was conducted using a custom-designed microfluidic device with embedded metal electrodes to apply mild electric field $(8 \pm 0.06 \mathrm{mV} /$ $\mathrm{mm}$ ) stimuli to hMSc/C2C12 coculture as well as monoculture. The cell viability together with gene and protein expression analysis allowed us to understand the cell fate in their proliferative/differentiative condition. The cell functionality and differentiation status of the cocultured cells are analyzed in the following sections.

\subsection{Cell morphology analysis}

The double lineage cocultured cells were channelized with the exogenous electric field. The influence of electric field on the cell morphology is illustrated in Fig. 2. Fig. 2a shows the cells, grown inside the channels with and without the electric field, on day 5 and day 9. The cells of different species/origin have adapted in the culture environment, without any biological discrimination or colonisation (Fig. 2b). The cells also exhibited regular morphological features, while physically communicating with each other.

\subsection{WST-1 assay for cell viability}

The cellular differentiation is regulated by the microenvironment, which modulates the genetic programming and alters the cellular fate. In order to assess any change in proliferation ststus quantitatively, we analyzed the cell samples at day 3, day 6 and day 9. The control sample was grown without electric field as a reference. The LOC device and chosen EF stimulation protocol supported cellular growth with a 

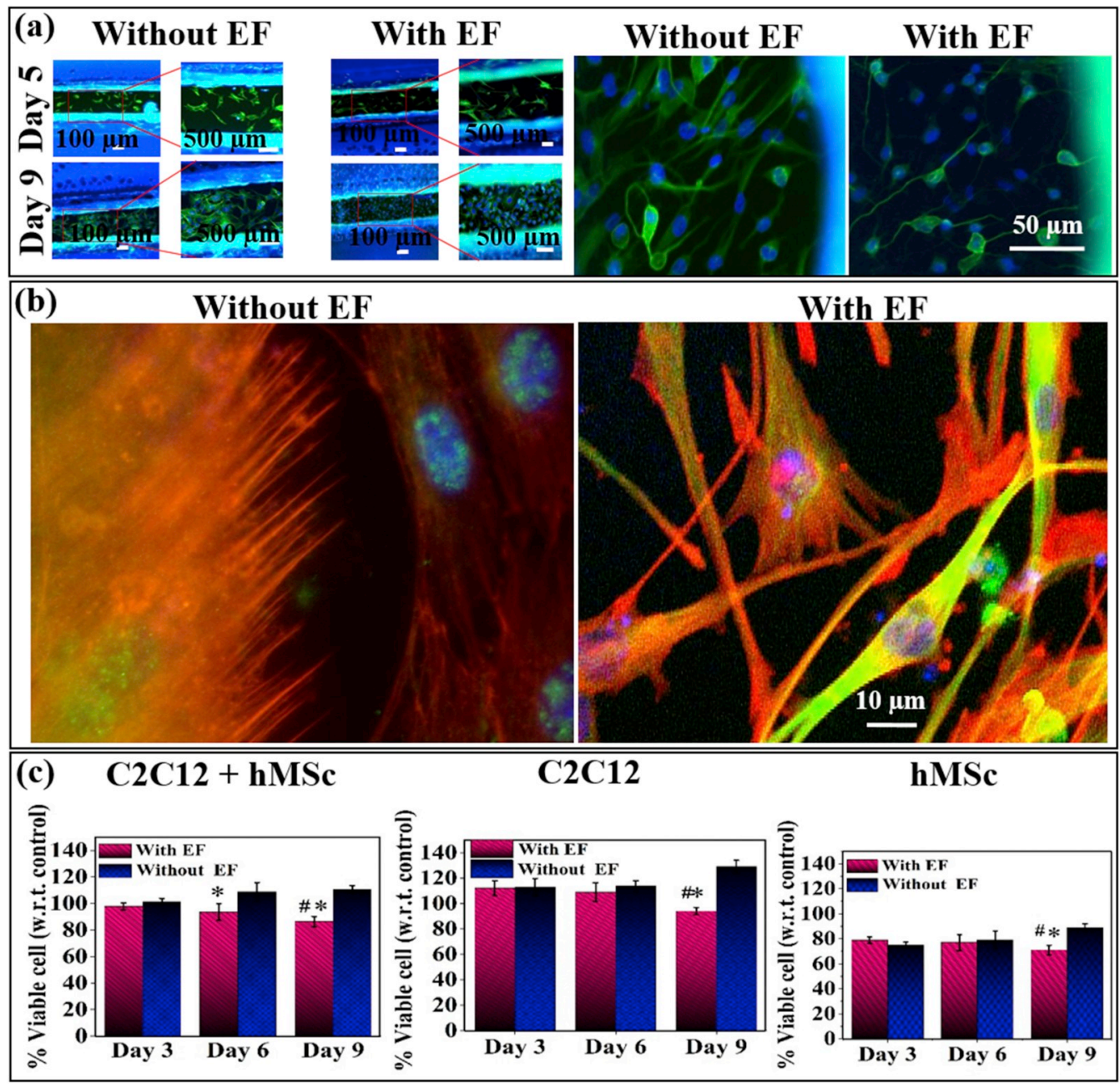

Fig. 2. Cytocompatibility results to confirm the cell status in the adopted electrical stimulation in PMMA based lab-on-a-chip device. Cytocompatibility of the PMMA based LOC device (a) Growth of the cells inside the channels used for differentiation of hMScs/C2C12 cocultures. (b) hMSc and C2C12 cellular tolerance to eachother for both electric field unexposed and exposed coculture. (c) The plots of WST-1 assay exhibit measured percentages of cell proliferation for day 3 , day 6 and day 9 occurred in the differentiation culture conditions for three sets of experiments (coculture, C2C12 monoculture, hMSc monoculture).* indicates statistically significant difference $(\mathrm{p}<0.05)$ of electric field treated sample w.r.t. without electric field. \# indicates statistically significant difference $(\mathrm{p}<0.05)$ of the samples w.r.t. control. considerable amount of viable cells. The signatures of the two inversely related processes (differentiation and proliferation) are reflected in the cell viability values (Fig. 2c). The maximum cell viability was found for the cells grown without electric field stimulation for upto day 9. The percentage of viable cells does not exhibit any statistical difference on day 3 and day 6 , in both the cases. At day 9 in culture, the application of electric field decreased cell viability by $\sim 11 \%$ than the initial culture (day 3). In other cases, we found the viability values to be statistically insignificant w.r.t. each other. The above results together denote the onset of cellular differentiation. Without the electric field, the cells have manifested the signature of slow proliferation.

\subsection{Analysis of hMSc and C2C12 behaviour in monoculture in comparison to coculture}

A distinct morphological comparison could be noticed among the three sets of the cultured cells (coculture, C2C12 monoculture, hMSc monoculture) (Fig. 3). The cocultured cells exhibited neurite-like projections, when stimulated with the electric field (Fig. 3a). The effect of the applied electric field on proliferation and differentiation of cultured muscle cells was studied in $\mathrm{C} 2 \mathrm{C} 12$ monoculture (Fig. 3b). In the present study, the cells, exposed to electric field stimulation, exhibited syncytic structures (Fig. 3b). SEM images could reveal the formation of fused myotube structures on exposing them to electric field (see supplementary section, Fig. S3(a)). Interestingly, the electrical stimulation has facilitated alignment of $\mathrm{C} 2 \mathrm{C} 12$ cells. On the other hand, the monoculture without the electric field shows normal proliferation with appreciable cellular spreading (Fig. 3b). The WST-1 assay of C2C12 monoculture on day 9 could be observed with increased proliferation, when compared with the day 3 culture (14\%), for the cells, without exposure to electric field. On being stimulated with the electric field, the cells show decreased WST-1 reading (14\%), as they have undergone differentiation towards myotube like structures (Fig. 2c). This has been confirmed with myogenin expression (Fig. S3(b)).

Upon the application of the electric field, hMSc monoculture started to grow in clustered structures (Fig. 3c, Fig. S4). To rule out the possibility that these structures were not merely formed with the debris of dead cells, we performed FDA/PI based live-dead assay (Fig. S4(b)) and WST-1 assay (Fig. 2c). The obtained staining pattern with FDA/PI showed sparsely dying core for most of the clusters together with a few of them with dying edge (Fig. S4(b)). The WST-1 assay was also not found to disregard the FDA/PI staining results, with well-established viability for the clusters.

We observed many of the clusters floating with a low degree of adhesion with LOC device substrate. Those were found to be smaller in size ( $\leq 100 \mu \mathrm{m}$ in diameter). Some of the clusters with a large diameter ( $\geq 500 \mu \mathrm{m}$ ), were also observed and those mostly adhered firmly to the substrate (Fig. S4(a)). Consequently, the cells were seen to be grown with elongated processes. The clusters exhibited positive expression for neural stem cell marker, SOX2 (Fig. S4(c)) along with neural markers (section 3.4). The expression of these marker proteins has enabled to characterize cell colonies as the neurosphere-like clusters. 

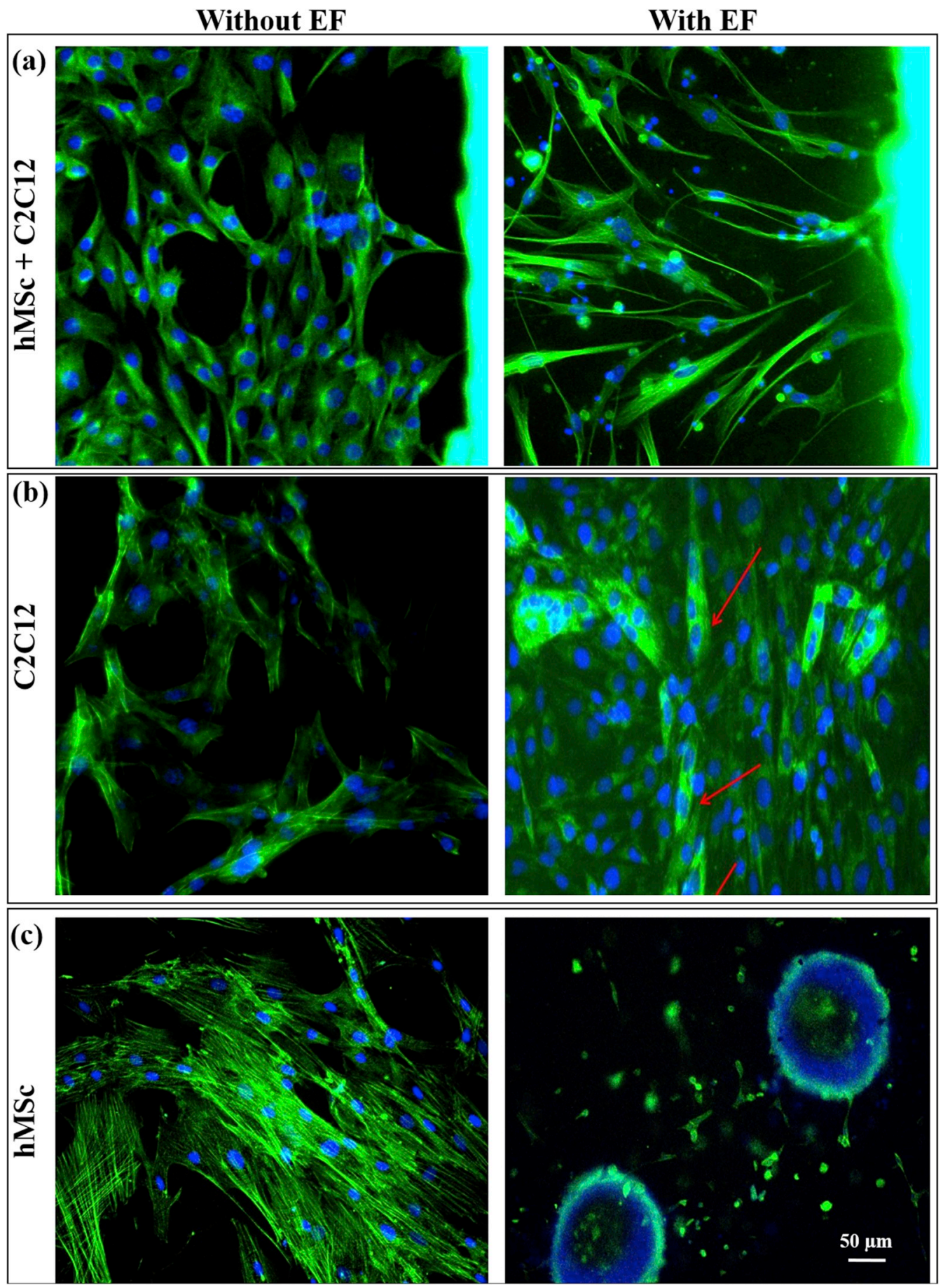

Fig. 3. EF stimulation aleters the cell morphological features of $\mathrm{C} 2 \mathrm{C} 12 / \mathrm{hMSc}$ coculture, $\mathrm{C} 2 \mathrm{C} 12$ monoculture, hMSc monoculture. (a) Change of cellular morphology of the electric field stricken cells in coculture is associated with cellular elongated processes whereas unexposed cells show lack of altered cell phenotype. (b) In monoculture of $\mathrm{C} 2 \mathrm{C} 12$, electric field stimulated cells show formation of syncytic structures. (c) hMSc monoculture, cells on application of electric field, forms clusters with growing outwardly proliferating edges. The hMSc cells growing without electric field are devoid of such cluster formation.

\subsection{Expression of neural markers proteins: induction, commitment and} maintenance of neural differentiation and architecture in the coculture

Next, we have validated the above observations using immunocytochemistry (Figs. 4-6). It is inevitable to validate the morphological change with expression of neural/neurogenic markers. We have emphasized on the expression of three protein markers, namely nestin (Fig. 4), an early neuronal marker; BIII-tubulin (Fig. 5), a marker expressed in immature neurons and MAP2 (Fig. 6), a marker expressed by all classes of immature and mature neurons. Nestin and $\beta I I I-t u b u l i n$ were assessed for all the three experimental groups (coculture, $\mathrm{C} 2 \mathrm{C} 12$ monoculture, hMSc monoculture). MAP2 has been characterised only for the cocultured cells as a confirmatory test along with PCR.

Fig. $4 a$ is the representative image of immuno-stained cells from three experimental sets for nestin which has been performed on the day 5. Minimal immuno-reactivity of the nestin was detected in the control cells, grown without electric field. In contrast, a good amount of nestin is localized in the cytoskeleton of the electric field stimulated cells cocultured cells. Also, it is worthwhile to mention that the actin filaments were clearly observed in the control (un-or-less-differentiated 


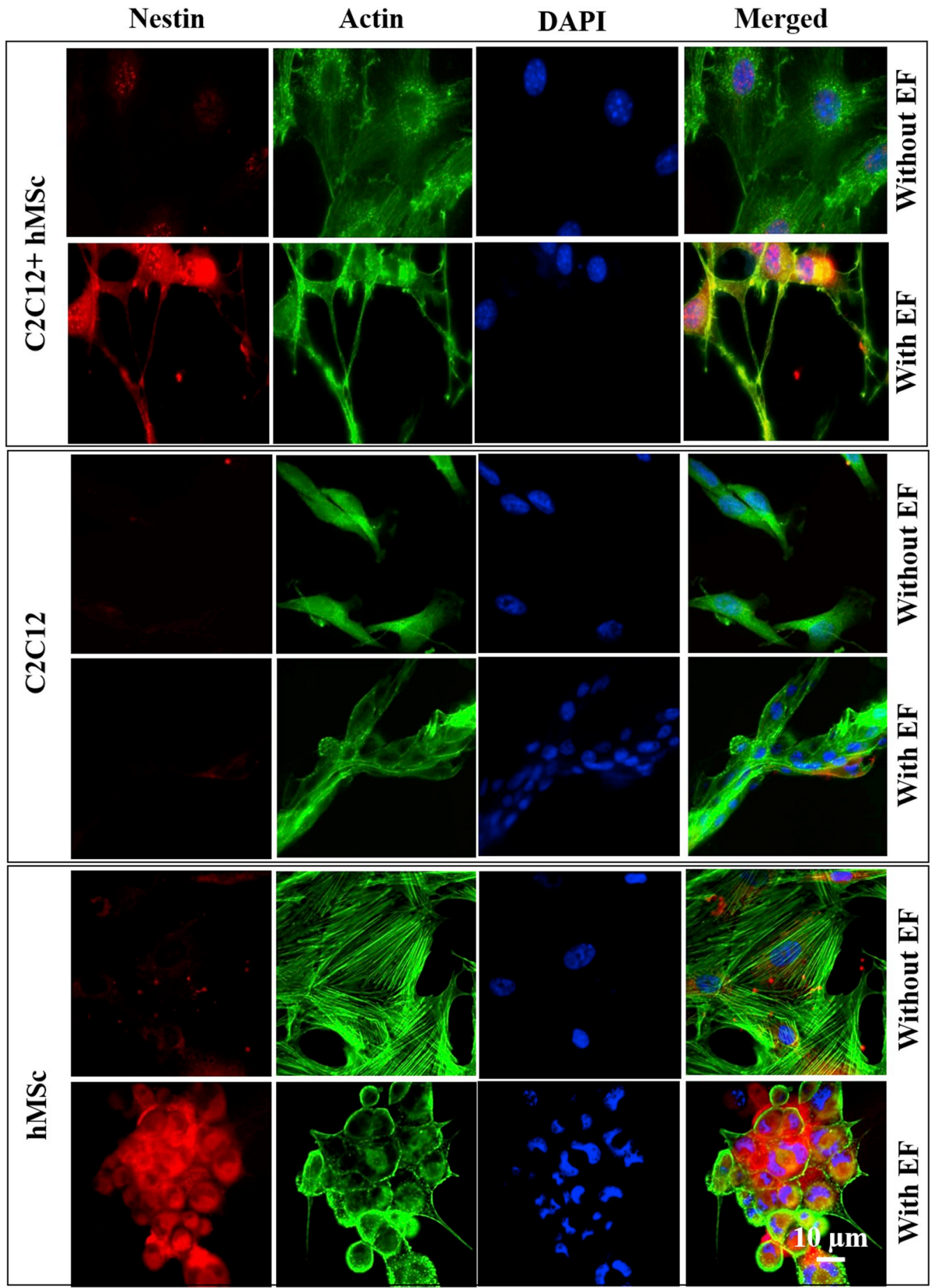

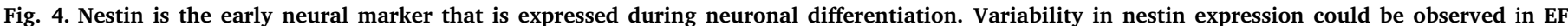



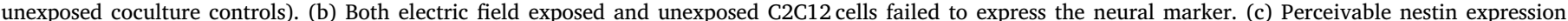
observed in the hMSc clusters, which was negative in unexposed cells.

cells). But, the actins were obscured due to the significant expression of the nestin (intermediate filament protein) in case of the electric field stricken cells. The neurosphere-like clusters, formed by monoculture of hMSc on exposure to electric field, has also expressed noticeable amount of nestin; whereas the electric field exposed C2C12 monoculture has failed to express the marker.

Fig. 5 depicts the expression level of $\beta$ III-tubulin on day 9, which is commonly found in the neural committed cells. Concomitantly, the cells stimulated with the electric field have a larger proportion of the cells showing BIII-tubulin positivity than the control. Furthermore, it could be noticed that $\beta$ III-tubulin proteins were localized in regions of the cells, mainly undergoing cytoplasmic extensions. This corroborates with the findings of Thrivikraman et al. who reported more positive BIII-tubulin expression for such neurite outgrowth [38]. On the other hand, the hMSc monoculture clusters, formed due to application of the electric field, has been stained positive for BIII-tubulin marker. Unlike the hMSc monoculture and coculture cells, the $\mathrm{C} 2 \mathrm{C} 12$ monoculture has not expressed measurable ßIII-tubulin, even after electric field 

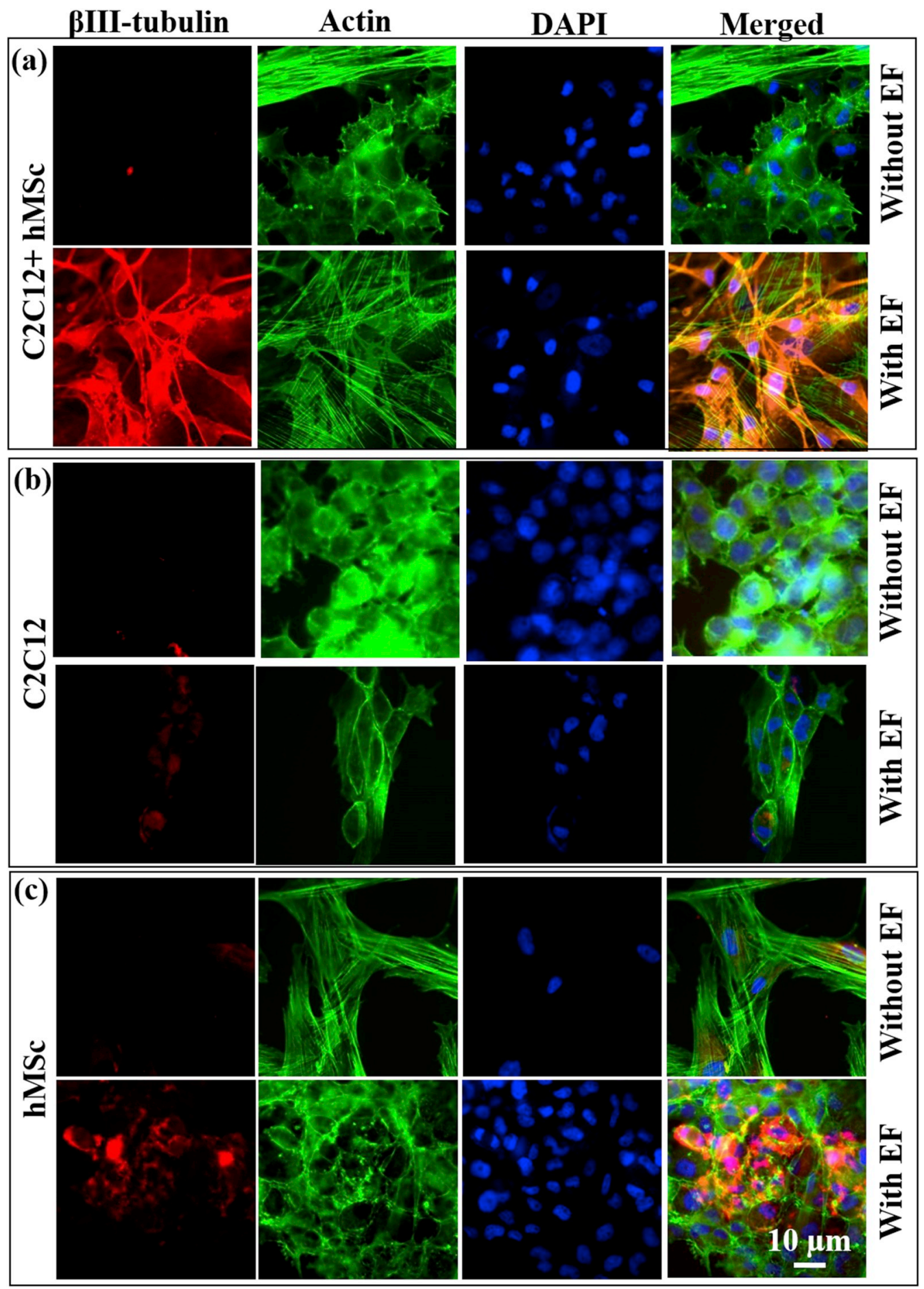

Fig. 5. Differential marker expression ( $\beta$ III-tubulin, actin, DAPI) on day 5 , reveal s signatures of outset of differentiation. (a) hMSc/C2C12 cocultured cells exhibited neurite-like projections with appreciable expression of BIII-tubulin, after the cells were exposed to electric field (absent in unexposed coculture cells). (b) Neural marker was absent inboth electric field exposed and unexposed C2C12 monoculture. (c) Considerable amount of BIII-tubulinhas been expressed for hMSc clusters, while the unexposed cells have failed to exhibit the same.

\section{application.}

MAP2 localization in the matured neurons mainly occurred in the neurites. The cell samples were collected on day 7 (image not included) and day 9 (Fig. 6) due to the late expression of MAP2. Such observation is in line with the previous observations, where the coculture cells, exposed to electric field, were able to express the markers in the neurite-like protruded regions (white arrows in Fig. 6a and b). On the contrary, the electric field unexposed cells have failed to express MAP2 protein.
The population of cells expressing these three vital neural specific markers (nestin/ßIII-tubulin/MAP2) were estimated roughly by counting the positively expressed cells in randomly chosen 10 fields. Such analysis reveals that highest expressing marker is the nestin, which is followed by the $\beta$ III-tubulin, scoring around 33\% lesser than the nestin. The MAP2 has a low expression in the cell population, only resulting about $25 \%$ positive cells. 


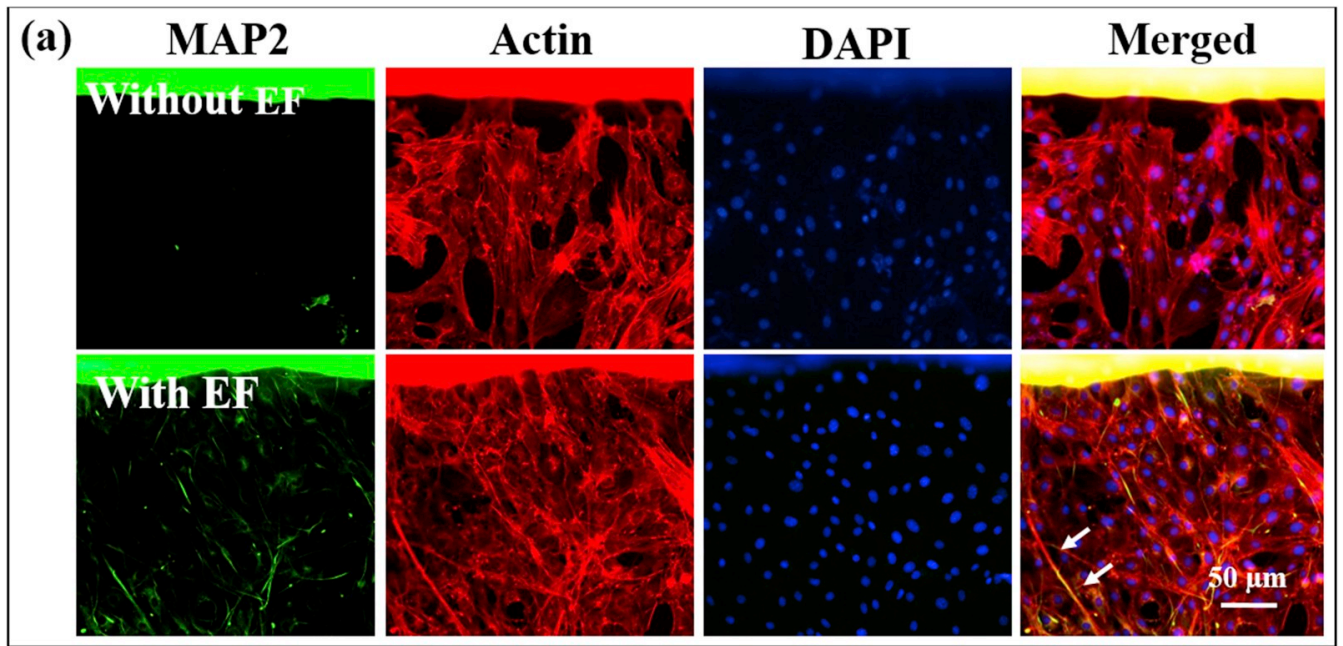

(b) MAP2
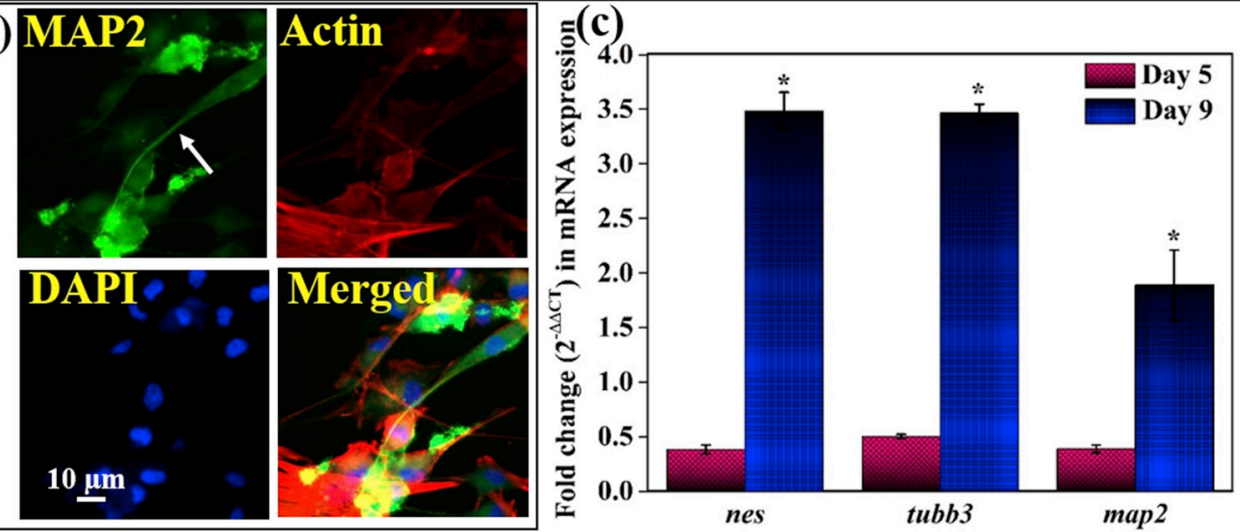

Fig. 6. Coculturing of multiple cell types is advantageous towards creating favourable cellular microenvironment to facilitate cell-cell interactions, leading to transdifferentiation. The present experimental condition enables the cocultured cells, expressing neural markers, like MAP2. (a), (b) Positive expression of MAP2 on day $9 \mathrm{~b}$ y the cells, reveals neural commitment. White arrows emphasises the expression of the MAP2 in the neurite-like structures. MAP2 seen to be well expressed on day 9 as a consequence of electric field trigger. (c) Quantitative RT-PCR analysis of neural differentiation genes. The mRNA expression levels of nes, tubb3 and map2 were normalized to the expression level of GAPDH. Data shown here are the $2^{-\Delta \Delta \mathrm{Ct}}$ values of the targeted genes. Three samples were used for each experiment and mean \pm SE were plotted. All the neurogenic genes have increased expression due to differentiation. The electric field stricken cells exhibit consistently higher expression of the neurogenic genes. * indicates statistically significant difference $(\mathrm{p}<0.05)$ of electric field applied sample w.r.t. day 3 .
3.5. Gene expression analysis of neuron-specific markers (quantitative PCR for $m R N A$ detection)

Quantitative PCR is widely regarded as the confirmatory test for the above discussed phenotypic characterization by immunostaining. Accordingly, mRNA expression of some of the specific genes were analyzed for day 3 and day 9 to assess the neural commitment (Fig. 6c). The bargraphs in Fig. 6c show fold change of the gene expression of neural-specific mRNA in terms of $2^{-\Delta \Delta C T}$ value with respect to the electric field unexposed cells of the same day (refer to table: S1, S2, S3 of annexure). An initial level of neural differentiation was confirmed by expression of nestin gene (nes). The fold change in nes mRNA on day 9 with electric field, compared to that of the control of the same day, scored $\sim 3.5$ fold increase, which could be considered as a signature of neural induction. For the observation on day 3, the expression of nes mRNA resulted quite low, which is only $\sim 0.4$ fold with respect to the unexposed cells.

The neural tubulin-III gene (tubb3) encodes for a class III member of the beta tubulin protein family. The heterodimer of the microtubule is the assemblage of $\beta$-tubulins and $\alpha$-tubulin. In case of neural cells, class-III member of the $\beta$-tubulin serves as the subunit of the microtubules. This protein is primarily expressed in neurons and may be involved in neurogenesis. The mRNA expression corresponding to $\beta$ tubulin of the coculture on day 3 with electric field stimulation has been observed with the noticeable increase. On day 3 , the electric field stricken cells were able to express $\sim 0.45$ fold higher gene expression than the unexposed cells. This has been increased to as high as $\sim 3.4$ fold change on day 9 , compared to that without electric field.

The third gene targeted was the map2, whose expression pattern commensurates with the trend of other neural genes. Similarly, the electric field exposed cells were able to elicit more expressed mRNA in comparison to the control or without electric field for both the observations. Importantly, the map2 expression was quite lower than other genes. This could be because of the fact that the appearance of this protein occurs once the neural cell starts to mature. In the first observation on day 3 , the fold change in map 2 mRNA manifested statistically insignificant expression for the electric field exposed cells, which is only $\sim 0.4$ fold higher with respect to the unexposed cells. The expression succeeded further with a drastic increase in mRNA amount on day 9 with $\sim 2$ fold change with respect to the unexposed cells.

\subsection{FACS analysis for quantitative determination of cell differentiation}

The monoculture of $\mathrm{C} 2 \mathrm{C} 12$, upon exposure to the electric field, exhibited BIII-tubulin expression very less, which has scored only $\sim 27 \%$. Also, the hMSc/C2C12 coculture cells exhibited $\sim 46 \% \beta$ III-tubulin expression, under the electric field stimulation protocol (Fig. S5). Nevertheless, the hMSc monoculture also has achieved slightly lesser percentage of $\beta$ III-tubulin, expressing cells of about $\sim 37 \%$. On applying electric field along with coculturing, the $\mathrm{C} 2 \mathrm{C} 12$ cell achieved almost $\sim 72 \%$ of increase in the neural marker expression. The multipotent stem cells also attained an efficacy of increased marker expression of $\sim 21 \%$, when they were cocultured with $\mathrm{C} 2 \mathrm{C} 12$ under electric field stimulation. It is worthwhile to mention that the presently used culture protocol shows a good promise towards transdifferentiation of committed lineages.

\subsection{Physiological analysis of cocultured cells}

The confirmatory evidences of the neuron-like functionality of the cocultured cells were inferred by the intracellular calcium imaging and patch-clamp study. 



(c)

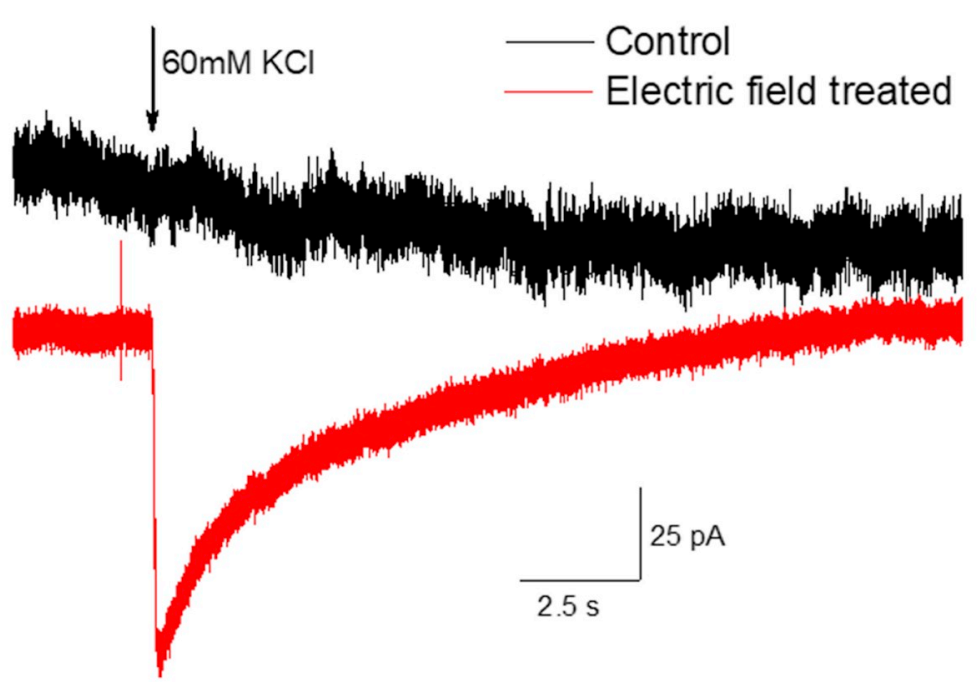

Fig. 7. Functional characterization using patch-clamp amplifier provides confirmatory evidence for the differentiation of hMSc/C2C12 coculture towards neural-like cells (a) Electric field treated cells in coculture exhibit intracellular calcium increase upon puff application of $60 \mathrm{mM} \mathrm{KCl}$ for $25 \mathrm{~s}$, whereas no relative increase in Fluo4 intensity was observed in the control cultures. (b) Intensity map of fluorescence imaging in real-time exhibit calcium response of control (upper panel) and electrical field treated cocultured cells (lower panel) upon $\mathrm{KCl}$ depolarization. (c) The representative electrophysiological recording using whole cell patch clamp technique show $\mathrm{KCl}$ induced inward current in electrical field stimulated cells $(5 / 5)$, but not in untreated controls.

\subsubsection{Depolarization of the neural-like cells}

3.7.1.1. Real time $\left[\mathrm{Ca}^{2+}\right]_{i}$ fluorescence imaging. Localized puff stimulation of Fluo4 loaded cells with $60 \mathrm{mM} \mathrm{KCl}$ for $25 \mathrm{~s}$ using a glass micropipette of $1 \mathrm{M} \Omega$ resistance and 5 psi pressure showed an increase in intracellular calcium in electric field stimulated cells (Fig. 7a). However, it has failed to exhibit any change in fluorescence intensity in untreated control. This observation is consistent with the excitable nature of neurons due to the presence of voltage gated channels. As expected, non-neuronal cells mostly exhibited nonexcitable behaviour and failed to show membrane depolarization upon high $\mathrm{KCl}$ application. Fig. $7 \mathrm{~b}$ shows the real-time data plotted as intensity map, depicting depolarization induced increase in intracellular calcium in the cocultured cells (video file: Control_KCl and Experimental_KCl).

Supplementary video related to this article can be found at https:// doi.org/10.1016/j.biomaterials.2019.119522

3.7.1.2. Whole cell voltage clamp recording. To further test the excitability of the electrical field stimulated cells, we measured $\mathrm{KCl}$ depolarization induced inward current. Previous reports have suggested that $\mathrm{KCl}$ depolarization is a measure of excitability [39]. Whole cell voltage clamp recordings showed strong inward current in electrical field stimulated cells with neurite-like protrusions upon puff application of $60 \mathrm{mM} \mathrm{KCl}$. However, it has failed to exhibit any 


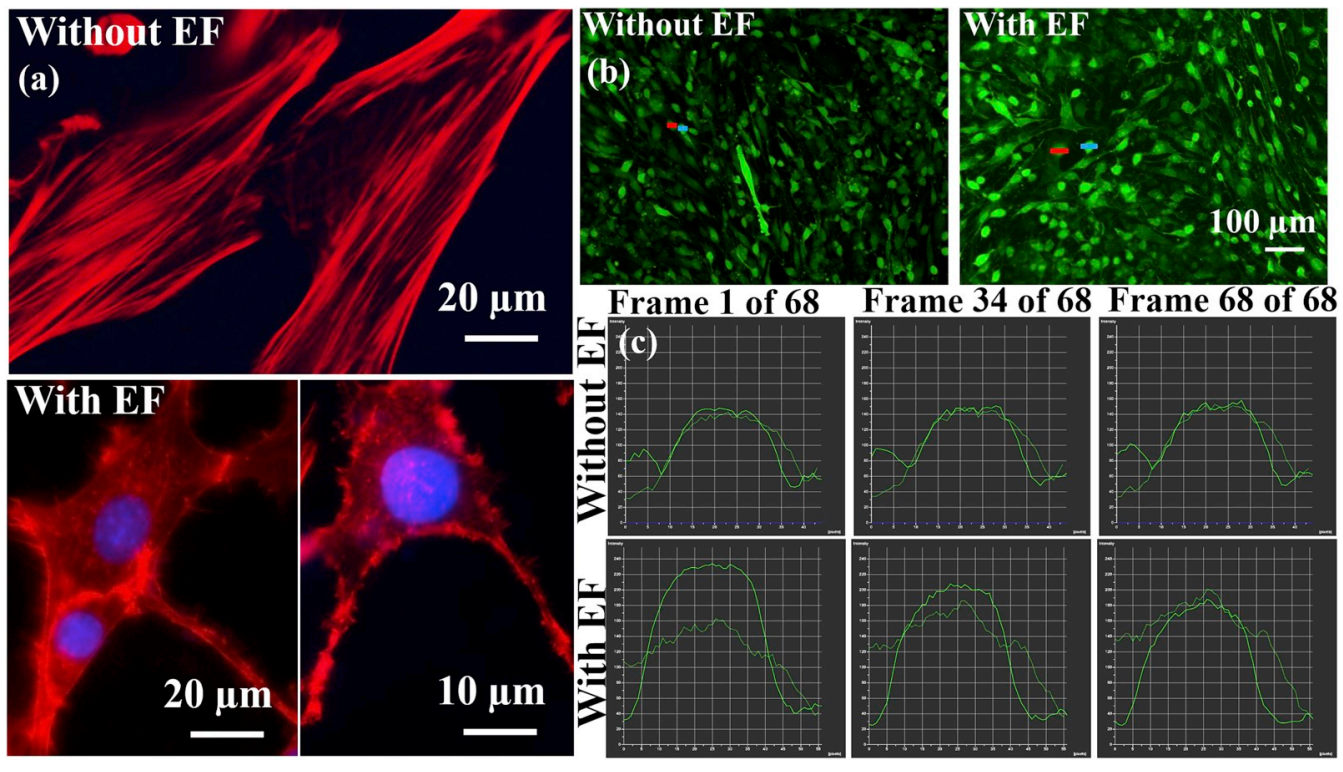

Fig. 8. Calcium $\left(\mathrm{Ca}^{2+}\right)$ signalling has significant roles in neurogenesis. Calcium oscillation acts as an attractor of cell differentiation. (a) Roughening of the cell-margin of the differentiated cell resembles neuronal spine formation previous to sprouting, which is absent for controls. (b) Fluo4AM staining of intracellular calcium exhibit oscillatory behaviour on exposure to electric field. Blue and red bar denotes the two selected cells exhibiting calcium transition in electric field exposed cells. They were used for plotting the pixel intensity. (c) Semiquantitative determination of the calcium oscillation of the two neighbouring cells (red and the blue mark in (b)). (see the video files: Control_CalciumOscillation and Experimental_CalciumOscillation). (For interpretation of the references to color in this figure legend, the reader is referred to the Web version of this article.) inward current in undifferentiated controls (Fig. 7c).

\subsubsection{Calcium oscillation: Functionality analysis with calcium imaging}

The electric field stricken cells were seen to have ruffle edges, along with a signature of neurite-like protrusions (Fig. 8a). The role of intracellular calcium oscillation has long been appreciated in regulating the cellular dynamics involved in the differentiation [40,41]. Interestingly, the calcium conduction between the intracellular to extracellular, subcellular to cellular or between two neighbouring cells are versatile and likely to depend on the type of biophysical cue.

The calcium dynamics in the hMSc and C2C12 cells were analyzed in cell samples on day 9. The profiles of calcium oscillation were recorded from the cells exposed to electric field, as shown in Fig. 8b and c (video file: Control_CalciumOscillation and Experimental_CalciumOscillation). The real-time recording shows the endogenous intracellular calcium oscillation in these cells, observed as change in pixel intensity. These changes in intracellular $\mathrm{Ca}^{2+}$ activity are attributed to the differentiation of hMSc/C2C12 cocultured cells to neural-like phenotype under electric field stimulation. Their minimal calcium activity has been seen to be increased due to the electric field stimulation.

\section{Discussion}

The present work demonstrates the impact of electric field stimulation and dynamic culture conditions on the transdifferentiation of coculture of $\mathrm{C} 2 \mathrm{C} 12$ and hMScs. It is important to mention that hMScs are capable to form neuron-like cells through the neurogenic differentiation pathway, though it was claimed by Ma et al. that hMScs could not be differentiated to form fully functional neurons [42-44]. Hence, the term neuron-like has been used in the present context to signify the potential of immature differentiation phenomenon. The process commences with the transformation of multipotent stem cell to committed neuroprogenitor-like cells. This requires the interplay of multiple factors, which regulates the shape of the stem cells for neural differentiation. Any change in the shape of the cells can develop intracellular tension. The application of electric field generates cellular tension, mediated by the cytoskeleton organization. This results in the forceful alteration of the cellular decision, leading to the induction of mechanosensitive signal pathway towards neural differentiation [45]. The above observations establish the apparent changes in cell morphology, characterised by thin extended axon-like protrusions. In this context, we first analyse the relevance of the electric field stimulation and examine the novelty of our culture protocol in the backdrop of earlier studies involving such biophysical stimulation.

\subsection{Rationale of electric field stimulation-based culture protocol}

The application of electric field stimulation can modulate cell functionality to different extent or through different pathways. Depending on the cell type and culture condition, the electric field could be applied intermittently as well as continuously. For example, Jain et al. demonstrated that continuous application of the low electric field of $<25 \mathrm{mV} / \mathrm{mm}$ for $6 \mathrm{~h}$ to the neuroblastoma cell line (N2a cells) could elongate the neurite effectively [46]. The relationship between the specific electric field and to the stem cell response cannot be generalized as the effect is quite diverse. The impact of such stimulation would depend on the cellular microenvironment, effect of cocultured cells and mode of application. Usually, the intermittent electric field application can be programmed with relatively lower electric field intensity. Thrivikraman et al. followed the protocol of exposing the cells in the electric field of $100 \mathrm{mV} / \mathrm{cm}$ for $15 \mathrm{~min} /$ day in combination with intracellular and extracellular nano-manipulators in the form of Aunanoparticles to trigger neurogenic/cardiomyogenic differentiation in hMScs [38]. In another study, it has been noticed that upon exposure of the hMSc to DC electric field of $100 \mathrm{mV} / \mathrm{cm}$ for $10 \mathrm{~min} /$ day, the cells exhibit neurogenic differentiation on PANI substrates [47]. Another mode of application is to give a short duration pulse of the relatively higher electric field. For example, Mobini et al. used DC electrical stimulation of $10 \mathrm{~V} / \mathrm{cm}$ electric field for $6 \mathrm{~h}$ to the adipose-derived mesenchymal stem cell cultures. This culture protocol guided them towards osteogenic differentiation. Zhao et al. reported that a $115 \mathrm{~V} / \mathrm{m}$ (DC) electric field for $2 \mathrm{~h} /$ day can induce directional migration and differentiation of neural precursor cells (NPCs) after culturing for 3 days [48]. Hammerick et al. showed that the application of electrical stimulation of physiological nature $(1 \mathrm{~V} / \mathrm{mm})$ and supra-physiological nature $(6 \mathrm{~V} / \mathrm{mm}$ and $10 \mathrm{~V} / \mathrm{mm})$ for short duration of $6 \mathrm{~h}$ facilitated galvanotaxis of the murine adipose-derived stromal cells [49]. In this context, the physiological range of electric fields occurring endogenously in the animal tissues has a strength as large as $2 \mathrm{~V} / \mathrm{cm}$. This also represents the upper limit of the electrical activities found at a wound site [50]. Fröhlich et al. reported that a low endogenous electric field of $2.29 \pm 0.27 \mathrm{mV} / \mathrm{mm}$ can guide the neocortical network formation [51]. In the above backdrop, the present experiments were 
conducted with electric field strength of $8 \pm 0.06 \mathrm{mV} / \mathrm{mm}$.

A few attempts are also being made to explore differentiation of stem cells in conditioned media, without biophysical cues. The adult human brain harbours neural stem cell (NSC) at the anterior sub-ventricular zone (SVZ) of the forebrain and hippocampus, which shows oligopotency towards neural lineages, like glial cells and functional neurons [52]. The stem cells migrate to the injured brain area to provide repairing functions. Comparing the hNSc and hESC, the hMScs are most widely investigated, because it has enormous potential to recreate the multiple lineages. MSCs have been transplanted in rodent models of stroke, brain, and spinal cord trauma and Parkinson's disease [53-55]. These treated animals have survived, with functional recovery. However, there are certain controversies regarding differentiation potential of stem cells with mesenchymal origin into neurons and glia, as they are ectoderm originated [56]. But it has been reported that the mesenchymal cells have been transformed into neurons. For example, Snyder et al. reported that on transplanting them into mouse brain, hMScs retained their capability to differentiate into neuronal and glial cells, in vivo [57]. Thrivikraman et al. have established the ability of the hMSc to differentiate into neuron-like lineage with upgradation of nestin, $\beta I I I-t u b u l i n$, like early neuron-specific markers [58]. It is worthwhile to mention that several other studies have established the event of neurallike differentiation of hMSc.

Some biomaterials substances have proven to be a good choice for differentiation of hMScs into neurons. For example, Yim et al. demonstrated the differentiation of hMScs towards neurogenesis by regulation of the morphology using micropatterned surfaces [59]. Her et al. created a 3D-environment with varying substrate stiffness to differentiate the hMSc into the neural lineage [60]. Some studies are also been conducted to reveal the capacity of the hMScs to differentiate towards a specific type of neuron. For example, Singh et al. transformed the hMSc cells into dopaminergic neurons [61]. Danielyan et al. reported the cholinergic neural-like differentiation of hMScs under the influence of erythropoietin [62]. The manipulation of cell-surface interaction by silicon nanowires facilitates the differentiation of hMScs into neurallike lineage, are reported by Kim et al. [63].

In the present context, it should be emphasized that the specific use of in-built electrodes within a microfluidic device allows us to create a uniform electric field distribution in the microfluidic culture (Fig. 1c and d). This has not been achieved before in earlier studies in the context of transdifferentiation. Also, the magnitude of electric field stimulation is fairly low and such a field is capable of guiding transdifferentiation of the volume of $\mathrm{C} 2 \mathrm{C} 12$ and hMScs.

\subsection{Establishing the hypothesis for the coculture differentiation}

\subsubsection{Exogenous and endogenous electric field interaction crossing the capacitive cell membrane}

It is important to know the magnitude of electric field experienced by cell coculture as the cells are shielded by the phospholipid membrane. This will help us to deduce the molecular pathway taken by the cells to respond to the applied stimuli. King et al. described the effective relationship between the endogenous and the exogenous field, while estimating the effective electric field reaching the cell interior [64]. The cell membrane, which has a very low conductivity, may or may not cause a drop in the electric field reaching inside the cells. Thus, it is very crucial to estimate the effective strength of electric field inside the cells, overcoming the capacitive barrier of the lipid membrane. The ratio of the electric field experienced inside the cell to that of the outside is,

$\frac{E_{2}}{E_{1}}=\frac{9 \eta}{\left(2+5 \eta+2 \eta^{2}\right)-2\left(1-2 \eta+\eta^{2}\right)(a / b)^{3}}$

where $\eta$ and a/b can be expanded as, $\eta=\frac{\sigma_{m}}{\sigma_{1}}$

$\left(\frac{a}{b}\right)^{3} \cong 1+\frac{3 \delta}{b}$

where, $\delta$ is the thickness of the cell membrane, a is the outer radius, $b$ is the inner radius of the cells, $\sigma_{\mathrm{m}}$ and $\sigma_{1}$ are the conductivity of the cell membrane and the extracellular saline fluid, respectively, the extracellular field is $E_{1}$, and $E_{2}$ is the internal electric field.

In case of conducting tissue, like heart, muscle, brain, the conductivity is about $\sigma_{1} \sim 150 \mathrm{~S} / \mathrm{m}$. Also, on the basis of the above formulation, it could be inferred that during the exposure of low-frequency electric field, the spherical cells experience a large drop in the electric field reaching inside the cell $\left(E_{2} / E_{1}=6 \times 10^{-4}\right)$. In contrast, the elongated cells (length $500 \mu \mathrm{m}$ ) with cylindrical shape, experience almost the same electric field as of the extracellular matrix $\left(E_{2} /\right.$ $E_{1}=0.99$ ).

The initial phase of cell differentiation starts with lack of electric field effect on the internal core of the cells. During this period, the surface receptors play a key role. Previous evidence suggests the presence of a membrane-mediated electric field response [65]. Once the cells are elongated, the core electric field experienced by the cells is close to the value of the extracellular electric field. This leads to direct effect of electric field on the intracellular region.

In the present case, initially, hMSc and C2C12 have a length of around $100-300 \mu \mathrm{m}$ and $60-90 \mu \mathrm{m}$, respectively. These cells experience negligible core electric field. Once the cells elongate to more than 500 $\mu \mathrm{m}$ along the axial direction, they started experiencing the core electric field, leading to intracellular electrocoupling activities. Moreover, C2C12, which are known to be multipotent/oligopotent stromal cells, receive paracrine signalling from the hMSc. This is elaborated further in the following discussion.

\subsubsection{Neural differentiation by electric field mediated changes in cellular microenvironment}

It is important to reiterate that the cells communicate initially with the electric field through the membrane receptors only. For example, Bezanilla et al. proposed that the electric field is sensed by the membrane protein by translocating the charges or by the movement of dipoles within the membrane [66]. The dislocation of charges and/or dipoles in an electric field produces electric current. In the case of the protein voltage sensors, the charges remain confined inside the cell membrane and keep moving within the protein [66]. Hence, the polarised proteins can modulate the cell behaviour. There is strong evidence, to suggest that a DC electric field can polarise a number of proteins. These include VEGF/EGF, acetyl choline receptors, receptors, and integrins [67-70]. These polarised surface receptors initiate an intracellular signalling cascade, mediated through src kinase (src), small GTPases, Phosphatase and tensin homolog (PTEN) and phosphoinositol kinase pathways [70,71].

In relation to the present study involving coculture with biophysical stimulations, the external field can also have electrophoretic and electroosmotic effects on the negatively charged proteins [72,73]. A DC electric field induces electrophoretic clustering of the membrane structures. For example, Lin et al. observed that mesenchymal stem cells experience the polarisation of lipid rafts towards the cathode after forming clusters [74]. The $\beta 1$-integrin molecules of the cell membrane along with some other known/unknown protein members form subsequent clusters or lipid rafts. The integrin/lipid raft complex is stabilized by Cav1, which is an integral inner membrane protein. This subsequently interacts with intracellular proteins, like RhoA, to activate signal transduction mechanism, through inactivation of p190 RhoGTPase [74].

Lim et al. mentioned that upon application of electric field, the hMScs can be guided along several differentiation pathways. They found that mesenchymal stem cells of human alveolar bone origin can 
undergo neurogenesis on reduced graphene oxide with the mediation of a pulsatile electric field [75]. They proposed that the cell-to-substratum adhesion machinery confers the regulatory effect on the neural differentiation, which is increased on graphene-coated surfaces. The neural processes, like axon and dendrons formation, are initiated as a result of regulated substrate adhesion. The focal adhesion kinase has a bidirectional role in neural commitment. Focal adhesion kinase (p125fak) has specific interaction with NCAM and the SRC-related tyrosine kinase, which promotes the growth of neural processes [76]. In contrast, FAK has also been described as the negative regulator of axon outgrowth by exerting pruning actions on the axons. This plays a sophisticated role in the axon dynamics [77]. In relation to electric field application, FAK exhibits altered expression and cellular distribution, which guides stem cell differentiation.

Another hypothesis is that electric field stimulation introduces conformational changes of the membrane proteins as the field-induced configuration gets trapped in local potential well. This may be less of thermodynamically favourable conformation than the native stable three-dimensional structure. For example, Aaron et al. suggested subsequent changes in the membrane protein conformation with downstream signal transduction, leading to genetic reprogramming [78]. In addition, the electrical stimulations are found to regulate the voltagesensitive ion channel activities with higher activation towards the cathode $[67,79]$. As the cells began to differentiate due to the cell surface mediated genetic reprogramming under the influence of extracellular electric field, the differentiated cells acquired elongated morphology. Such elongated morphology enables the cells to experience the intracellular electric field, which can influence cytoskeletal reorientation. For example, Li et al. provided evidence that the galvanic stress generates superoxides, which cause cytoskeletal reorganization through the activation of AKT/ERKs pathways [80].

The above pathway can be proposed for hMScs towards the neural differentiation under electric field stimulation. Nevertheless, the presence of the specialized media with B-27 and astrocytic conditioned medium has caused neural induction in the presence of electric field. This can be due to superficial membrane interaction through the membrane receptor. Alternatively, the selective electroporation of the membrane in the presence of electric field stimulation has allowed the signalling molecules to enter and to modulate the cell behaviour epigenetically.

\subsubsection{Induced neural differentiation of $\mathrm{C} 2 \mathrm{C} 12$ cells by the paracrine effect}

Several researchers reported the phenomenon of dedifferentiation of adult somatic cells (C2C12) into induced multipotent stem cells after treating them with transforming growth factor- $\beta(1)$ (TGF- $\beta(1)$ ). For example, $\mathrm{Mu}$ et al. demonstrated that a lower concentration of TGF$\beta(1)$ could promote $\mathrm{C} 2 \mathrm{C} 12$ myoblasts to express stem cell markers as well as to repress myogenic proteins, which stimulate dedifferentiation [81]. Watanabe et al. successfully modulated the C2C12 myoblasts into active neural phenotype by activation of REST/NRSF target genes. This allows the activation of terminal neuronal differentiation genes by overriding muscular differentiation [82]. Other than invasive genetic engineering procedures, small chemo-molecules can act as inducers of neuronal differentiation for $\mathrm{C} 2 \mathrm{C} 12$. For example, Williams et al. developed a protocol for $\mathrm{C} 2 \mathrm{C} 12$ (cocultured with $\mathrm{PC} 12$ ) to differentiate into neurons using neurodazine [83].

Williams et al. have reported the recycling of synaptic vesicle stained with FM1-43 dye at the exposure of high $\mathrm{K}^{+}$ions [84]. Zhao et al. found that an electric field strength of $150 \mathrm{mV} / \mathrm{mm}$ for $20 \mathrm{~h}$ has a stimulatory effect on the cell division by altering the cell division orientation. Titushkin et al. reported that the exposure of the cells for $60 \mathrm{~min}$ at $2 \mathrm{~V} / \mathrm{mm}$ electric field caused actin remodelling [85,86]. The intermittent resting time is important as the cells use that as a recovery period.

There is strong evidence that the hMScs were characterised as a good vehicle to deliver growth factor and micro-RNAs. For example,
Suzuki et al. used the transformed hMScs to modulate neuroprotection and neural proliferation of the rat adult muscle, in vivo. [87].

Also, hMScs are reported to be capable of modulating other cell lines by efficient exosome secretion [88]. Aaron et al. reported the autocrine and paracrine effect on the cells through the secretory growth factors [78]. Dabrowska et al. established the biological aspect of paracrine signalling of the hMSc to other cell types. They showed that hMScs, being multipotent cells are capable of influencing the organotypic hippocampal culture. The putative regenerative interactions among Wharton-jelly-isolated hMSc and organotropic-hippocampal cells mediate the molecular mechanisms mainly through mesenchymal cell paracrine activity [89]. This helps in creating the neuroprotective effect towards the primary cells of hippocampal culture. The secretion profiling of the spent media revealed that the hMScs are capable of releasing TGF $\beta$ (tumor necrosis growth factor $\beta$ ), IL-2 (interleukin-2), IL-10 (interleukin-10), FGF (fibroblast growth factor), and IGF (insulinlike growth factor) and VEGF (vascular endothelial growth factor) [89].

In the present work, it is hypothesized that the hMScs have mediated the $\mathrm{C} 2 \mathrm{C} 12$ based neurogenesis through secreted exosomes containing neurotrophic factors, endocytosed by the $\mathrm{C} 2 \mathrm{C} 12$ cells. The C2C12 cells were influenced and redirected towards neurogenesis, probably through activation of B-cell translocation gene 2 (btg2) [83]. Through the intracellular delivery of the exosomes, the cells receive the intact and functional miRNA and growth factors without any perturbation. Likewise, the low intensity constant electric field for a prolonged duration time mimics the physiological neurogenic condition. This commits the hMScs into neural differentiation, which in turn have cross-talk with the $\mathrm{C} 2 \mathrm{C} 12$ through paracrine signalling. This study also implies that $\mathrm{C} 2 \mathrm{C} 12$, being the stromal origin multipotent cells, can be transdifferentiated into neuron-like cells. As experimental evidence, the pattern bound cell culture of hMSc and C2C12 (supplementary information) shows induction of neurogenesis in the C2C12 cells, which were adjacent to the hMSc clusters (Fig. S6).

\subsection{Compatibility of heterogenic murine and human origin cells in a coculture}

Heterogenic coculture is noncanonical and difficult to establish, compared to homogenous culture. It is necessary to have cell-to-cell cross-talk and healthy growth pattern of the cells to have a synergistic output. Mesenchymal stromal cells, being the most potent cell type of regenerative cells, were chosen to exploit the extensive paracrine capability to modulate the functionality of other cell types. C2C12 cells were chosen to be modulated by the hMScs under the influence of the electric field.

Sassoli et al. mentioned that coculture of these two cells (hMSc and C2C12) under canonical culture system can potentially improve the ability for skeletal muscle repair/regeneration [90]. The crosstalk between the bone-marrow hMScs and C2C12 myoblast was established by analyzing the chemical communication between cells of two different origins. The secretion of vascular endothelial growth factor (VEGF) by the hMScs was thought to be the molecules responsible for such an outcome [90]. Ryu et al. made attempts to show that the C2C12 and hMScs, when layered together onto chitosan substrates, promote myogenesis [91]. The mouse embryonic stem cells were successfully transformed to hepatocyte-like cells by the liver nonparenchymal cell lines in the coculture [92]. Such cocultured cells can successfully exhibit the rare noncanonical differentiations.

In the above context, Fig. 2 reveals that hMScs and C2C12 cells were well maintained in the close vicinity without imposing any detrimental mutual exclusion effects. The cells could communicate extensively and there was no trace of colonization of same cells. 


\subsection{Expression of neural markers by cocultured cells under the influence of electric field}

Nestin is a neural progenitor-specific member of type IV intermediate filament (IF) proteins. It is known that nestin is specifically expressed in neural progenitors, but never in matured neurons and glial cells [93]. This plays a pivotal role in neural transdifferentiation in the present context. As a consequence of neural differentiation, the hMSc/ C2C12 coculture has been characterised with the expression of nestin. Unlike most other IF proteins, nestin cannot form stable filaments by itself, but can form co-polymer in vitro and in vivo by association with type III IF proteins, such as vimentin [94]. Chang et al. similarly found elevated expression of nestin in DC electric field stimulated cells [95]. Also, the same research group reported the associated expression of BIII-tubulin along with GFAP and O4. In corroboration with the reported marker expression study, it has been found that the cocultured cells have expressed $\beta$ III-tubulin as well, in the present case. The FACS analysis quantitatively establishes that a substantial percentage of cells was expressing $\beta$ III-tubulin (see the supplementary section, Fig. S4). Importantly, MAP2 is expressed as a compulsory neural marker after nestin expression. The electric field has been reported to have enhanced fibroblast growth factor receptor (FGF-R) and its ligand modulated signalling pathway [85]. As a consequence of electric field application, MAP2 may have been expressed by the action of FGF signalling pathway. Further signalling analysis by Chang et al. also revealed that FGF-2-induced nestin expression is mediated through FGFR-MAPK-ERK signalling axis and the transcriptional factor Sp1 [96]. In the present study, we found the NCAM silencing results in lack of neural morphology of the electric field exposed cells, when compared to the NCAM expressing cells (see the supplementary section, Fig. S7).

\subsection{Physiological changes provide confirmatory evidence towards neurogenesis in coculture}

\subsubsection{KCl mediated membrane depolarization}

A higher amount of extracellular potassium destabilizes the membrane potential of excitatory cells, thereby causing opening of voltage gated sodium and calcium ion channels. In addition to the calcium current observed in our patch clamp experiments, $\mathrm{KCl}$ depolarization of cocultured cells also showed bolus of calcium entry in electric field stimulated cultures (Fig. 7). This is a characteristic property of the excitable cells, thereby strengthening our claim of their similarity to neurons.

\subsubsection{Calcium oscillation}

When neural stem cells are exposed to electromagnetic stimulation, spontaneous $\mathrm{Ca}^{2+}$ transition occurs due to an increase in Cavl.2 ( $\mathrm{L}$ type) channel activity [97]. This stimulates neurogenesis by $\mathrm{Ca}^{2+}$ influx via L-type $\mathrm{Ca}^{2+}$ channel, consisting mainly of CREB phosphorylated transcription factor-mediated pathway. In particular, in the case of stimulated bone marrow-derived hMSc, increased levels of neuronal marker gene expression are associated with CREB-mediated phosphorylation [98]. It has been speculated that electric field exposure increases CREB to DNA binding activity, particularly for classes of nucleotides called cAMP [99].

In the present study, we have monitored the calcium oscillatory activity in $30 \%$ of the cells, which were specifically in physical contact with each other (Fig. 8). This process is important for many cellular phenomena, including cell proliferation, differentiation, signalling and other adaptive functions [100,101]. Electric field stimuli caused a dominant activity of L-type calcium channel along with the activation of $\mathrm{N}$-type calcium channels in neuronal gene expression. Nonetheless, the neurons can detect voltages with the help of two different classes of voltage-activated calcium channel and recruitment of certain intracellular signalling pathways. Other than the calcium channels, the formation of gap junctions as the representative of electric synapse can also mediate the movement of $\mathrm{Ca}^{2+}$. This is perhaps the possible reason for calcium oscillation in the physically communicating cells.

\section{6. $h M S c / C 2 C 12$ monoculture functionality under electric field stimulation}

The differences in cellular functionality are more evident when we analyse the baseline results of monocultures. Murine C2C12 myoblasts, being multipotent, have the ability to retain their differentiation potential, when grown under biophysical stimulation. The myoblasts fuse with neighbouring cells to form multinucleated myotubes [102]. Similar findings were also reported by Park et al. and they demonstrated that with electric field stimulation, the $\mathrm{C} 2 \mathrm{C} 12$ cells started to express the myotube maturation markers, such as myoD [25,103]. Thelen et al. reported that electrical stimulation at $3 \mathrm{~V} / \mathrm{cm}$ with $2 \mathrm{~Hz}$ enhances contractile properties in the C2C12 myotubes [104]. They even found expression of SERC $\mathrm{Ca}^{2+}$ type 1 proteins, which provide strong evidence for myogenic differentiation of the $\mathrm{C} 2 \mathrm{C} 12$ cells, when exposed to electrical stimuli. Tanaka et al. found the $\mathrm{C} 2 \mathrm{C} 12$ cells align on application of electric field [103]. In summary, the electrical cues cause the fusion and differentiation of the $\mathrm{C} 2 \mathrm{C} 12$ cells into multinucleated structure formation.

In monoculture, hMScs, which are known to have altered adhesion due to electric field application, form clusters (see the supplementary section, Fig. S4(a)). These neurosphere-like structures were the outcome of less adhesiveness of the hMScs, in comparison to the electrically stimulated $\mathrm{C} 2 \mathrm{C} 12$ which are capable of secreting collagen I (adhension protein) [25]. Moreover, the neurosphere formation is influenced in the presence of EGF [105]. The electric field stricken hMScs are known to secrete EGF and to create polarity [67]. The EGF is known to have positive influences in the formation of neurosphere [106]. The combinatorial effect of the above factors contributes to the formation of neurosphere-like clusters for electric field stimulated hMSc monocultures.

In particular, hMScs are capable of exerting a trophic effect on the neighbouring cell, which can alter the cellular behaviour. For example, in coculture pellets of hMSc and chondrocytes, it has been observed that due to the extended trophism of bone marrow stem cells, the chondrocytic differentiation was aggravated, irrespective of culture condition or cellular origin [107]. This implies that the paratrophic effect of the hMSc in the form of chemical cue or contact cue in cocultures is a general phenomenon with potential for redirecting the cells into the differentiative path along with increased cell growth.

The human mesenchymal stem cells, isolated from the umbilical cord and Wharton jelly, secrete activin A into the extracellular milieu, when they are cocultured with the neural stem cells isolated from subventricular zone (SVZ). It is also reported that the differentiating stem cell in neural path secretes exosome containing miRNA (miR125b), which can be endocytosed by other cells [108]. This further recruits the cells in the neurogenic path. For instance, the coculturing of neural stem cells (NScs) with hMSc can drive the former along the path of neurogenesis [109]. The electric field directed exocytosis by the stem cells plays the role of neighbouring cell recruitment. It is worth mentioning that the rapid loss of stemness of in vitro-isolated NScs possess a serious challenge for neural regeneration. During any injury of the nervous system, a limited population of the newly generated cells is recruited to the damage sites, and also takes part in neural repair [1]. Mesenchymal stem cells are promising cell sources for CNS regeneration due to their greater abundance and easier accessibility, as compared to neural stem cells and embryonic stem cells.

\subsection{Relevance in the context of cellular reprogramming}

There are no effective treatments for neurodegenerative diseases such as Alzheimer's disease (AD), Parkinson's disease (PD), Huntington's disease and amyotrophic lateral sclerosis. Such diseases could be well 

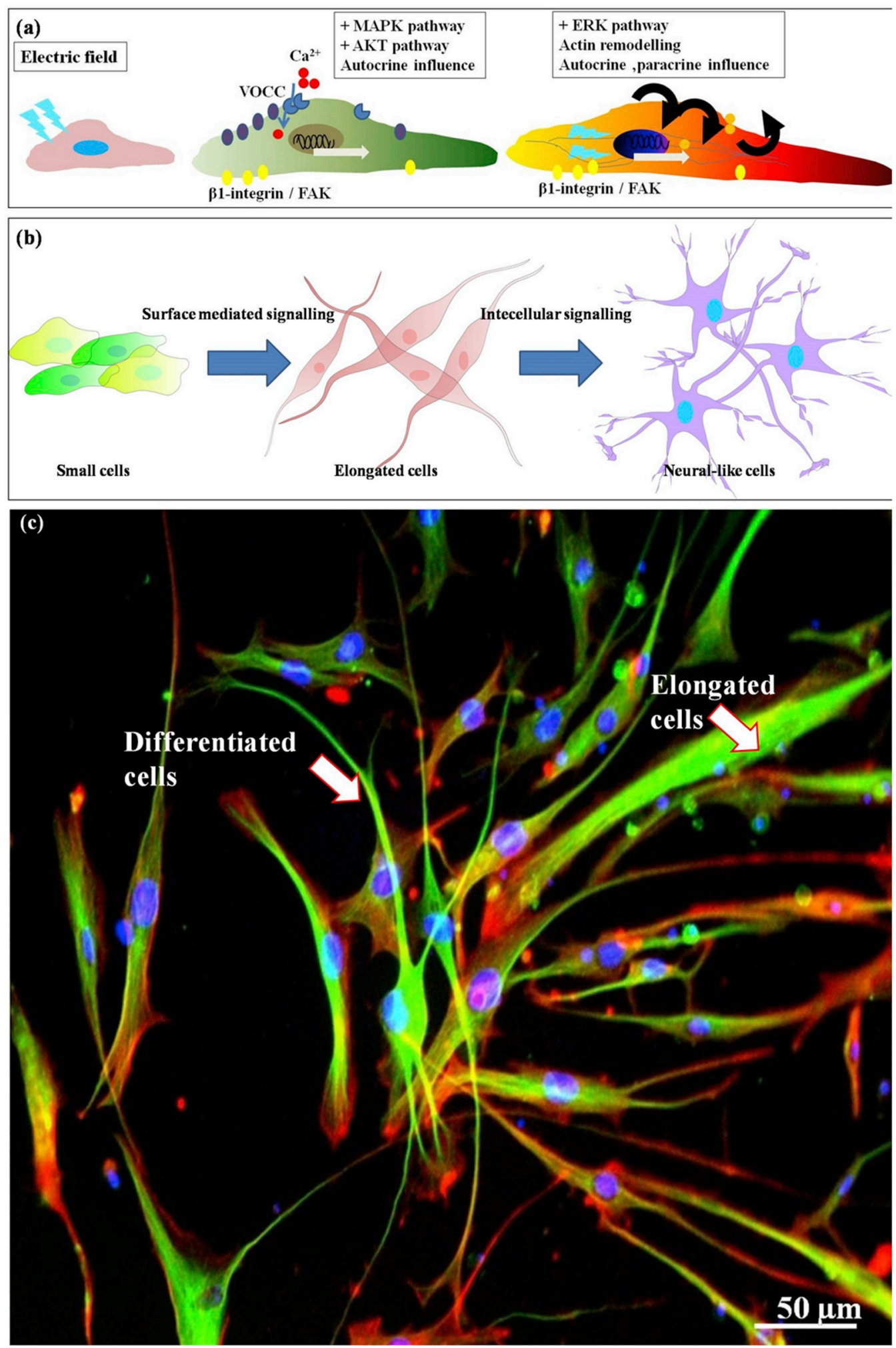

Fig. 9. The hypothesis for self-modulation of electric field stricken cells towards neural differentiation based on the cell-to-self autocrine and cellto-cell paracrine signalling, medicated via secreted growth factors. (a) Electric field stricken cells mediating the electric field through the cell surface sensors, initial elongation enables the cell to perform intracellular execution of electric field outcomes. The probable signalling are displayed against each step. (b) Schematic illustration view of the cocultured cells getting transdifferentiated to neurallike cells. (c) Immunofluorescence image with actin (red)/ $\alpha$-tubulin (green)/nucleus (blue) staining revealing elongated cell according to the hypothesis and cells with neurite-like projections. (For interpretation of the references to color in this figure legend, the reader is referred to the Web version of this article.) managed by introducing regenerative engineering approaches. This concept has been proposed to be more effective than the conventional drug therapy as well as invasive clinical approaches like deep brain stimulation $[110,111]$. The choice of the cells to perform such neural transdifferentiation for cell replacement therapy is unfortunately restricted to embryonic stem cell and induced pluripotent stem cell (iPSc). It is known that iPScs can be converted to DA neurons which restore phenotypes in animal models of Parkinson's disease [112]. iPScs respond well to a pattern of the interacting surfaces, which could divert the cells towards neurogenesis, along with the possibility of generating iPSC-derived nucleus pulposus (NP) cells in a mouse model in hypoxic condition [113,114]. Nevertheless, apart from the successful implementation of iPSc for reprogramming of cells towards neurogenesis, the present protocol reveals a new approach for transdifferentiation of non-neuronal cell (C2C12 (pre-myoblast) cells) to neural-like phenotype, when cocultured with hMScs under electric field stimulation. This 
essentially supports the hypothesis for transdifferentiation of somatic cells with stem-like properties (example C2C12) into neuron-like by an optimal microenvironment. In the backdrop of this hypothesis, it should be mentioned that hMScs, while undergoing differentiation in culture, can regulate the functionality of other cell types.

In the present context, the dedifferentiating and transdifferentiating potential of $\mathrm{C} 2 \mathrm{C} 12$ along with exploiting the stemness of hMSc, allows us to induce a sub-population of cells with characteristics of muscle stem cells into non-muscle lineage, through application of intermittent electric field (Fig. 9). This could be used for transmuting cellular functionality into generating autologous stem cells for regenerative medicine.

\section{Conclusions}

The following important conclusions emerge from this work.

(a) The present study demonstrates the unique ability of low-intensity electric field stimulation $(8 \pm 0.06 \mathrm{mV} / \mathrm{mm})$ towards transdifferentiation of $\mathrm{C} 2 \mathrm{C} 12$ myoblast cells to neural-like cells, when cocultured together with hMScs, in conditioned media within custommade PMMA microfluidic device. The novel protocol has facilitated the transdifferentiation of a non-stem cell type towards neuron-like cells under the influence of differentiating hMScs by electric field stimulation.

(b) The monocultured C2C12 cells have manifested cell fusion under the influence of similar culture conditions. On the other hand, the hMSc monocultures formed neurosphere-like clusters. Both the cell types have exhibited an altered phenotype, but failed to exhibit the level of neural-like morphology, in isolation.

(c) Statistically significant upregulation of neural markers in cocultured cells was recorded at protein and mRNA level. The calcium oscillation is clearly exhibited in the cocultured cell population. The paracrine signalling or the physical communication of the differentiated hMScs drives the C2C12 cells to undergo transdifferentiation towards neural-like morphology.

(d) The electrophysiological analysis of $\mathrm{Ca}^{2+}$ depolarization strengthens our finding of differentiation of $\mathrm{C} 2 \mathrm{C} 12$ cells to neurallike cells upon electrical stimulation. Further, our patch clamp study demonstrates an important characteristic of neural-like cells, significant intracellular calcium-signalling, in the electric field stimulated cocultured cells.

To conclude, biomicrofluidics based devices with tailored electric field stimulation allow us to study the aspect of transdifferentiation of stromal cells, cocultured with the mesenchymal stem cells. The coculturing of multiple cell lines can make difficult differentiation pathways realisable with the intervention of electric field stimulation.

\section{Acknowledgments}

The authors acknowledge the Department of Science and Technology (DST) through FIST and MathBio program and major funding support from Department of Biotechnology (DBT), Government of India via the 'Translational Centre on Biomaterials for Orthopaedic and Dental Applications' and 'Bioengineering Biodesign Initiative Phase 2' project. We are also thankful to the Biosystems Science and Engineering (BSSE), IISc for the immense financial and administrative support. We also mention the help of Dr. Uttara Chakraborty (Senior Research Scientist) for FACS. The authors are thankful to Zehra, Amit, Vaibhab for helping to perform the $\mathrm{KCl}$ stimulation experiments. Sharmistha is immensely grateful to Prerana, Soumitra, Deepa and Asish for their unconditional support, during revision of the manuscript.

\section{Appendix A. Supplementary data}

Supplementary data to this article can be found online at https:// doi.org/10.1016/j.biomaterials.2019.119522.

\section{References}

[1] G. Thrivikraman, S.K. Boda, B. Basu, Unraveling the mechanistic effects of electric field stimulation towards directing stem cell fate and function: A tissue engineering perspective, Biomaterials 150 (2018) 60-86.

[2] M.F. Pittenger, et al., Multilineage potential of adult human mesenchymal stem cells, Science 284 (5411) (1999) 143-147.

[3] J. Oswald, et al., Mesenchymal stem cells can be differentiated into endothelial cells in vitro, Stem Cells 22 (3) (2004) 377-384.

[4] S. Makino, et al., Cardiomyocytes can be generated from marrow stromal cells in vitro, J. Clin. Investig. 103 (5) (1999) 697.

[5] S. Snykers, et al., In vitro differentiation of embryonic and adult stem cells into hepatocytes: State of the art, Stem Cells 27 (3) (2009) 577-605.

[6] A. Arthur, et al., Adult human dental pulp stem cells differentiate toward functionally active neurons under appropriate environmental cues, Stem Cells 26 (7) (2008) 1787-1795.

[7] D.G. Phinney, D.J. Prockop, Concise review: Mesenchymal stem/multipotent stromal cells: The state of transdifferentiation and modes of tissue repair-current views, Stem Cells 25 (11) (2007) 2896-2902.

[8] R. Nuccitelli, A role for endogenous electric fields in wound healing, Curr. Top. Dev. Biol. 58 (2003) 1-26.

[9] S. Naskar, V. Kumaran, B. Basu, On the origin of shear stress induced myogenesis using PMMA based lab-on-chip, ACS Biomater. Sci. Eng. 3 (6) (2017) 1154-1171.

[10] H. Jahani, et al., Controlled surface morphology and hydrophilicity of polycaprolactone toward selective differentiation of mesenchymal stem cells to neural like cells, J. Biomed. Mater. Res. A 103 (5) (2015) 1875-1881.

[11] M. Yamada, et al., Controlled formation of heterotypic hepatic micro-organoids in anisotropic hydrogel microfibers for long-term preservation of liver-specific functions, Biomaterials 33 (33) (2012) 8304-8315.

[12] H.-C. Han, et al., Nano-textured fluidic biochip as biological filter for selective survival of neuronal cells, J. Biomed. Mater. Res. A 103 (6) (2015) 2015-2023.

[13] N. Nakajima, et al., MicroRNA-1 facilitates skeletal myogenic differentiation without affecting osteoblastic and adipogenic differentiation, Biochem. Biophys. Res. Biochem. Biophys. Res. Commun. 350 (4) (2006) 1006-1012.

[14] K. Yeow, et al., Inhibition of myogenesis enables adipogenic trans-differentiation in the C2C12 myogenic cell line, FEBS (Fed. Eur. Biochem. Soc.) Lett. 506 (2) (2001) 157-162.

[15] T. Schilling, et al., Plasticity in adipogenesis and osteogenesis of human mesenchymal stem cells, Mol. Cell. Endocrinol. 271 (1-2) (2007) 1-17.

[16] R. Nishimura, et al., Smad 5 and DPC4 are key molecules in mediating BMP-2induced osteoblastic differentiation of the pluripotent mesenchymal precursor cell line C2C12, J. Biol. Chem. 273 (4) (1998) 1872-1879.

[17] S. Dong, et al., MicroRNAs regulate osteogenesis and chondrogenesis, Biochem. Biophys. Res. Commun. 418 (4) (2012) 587-591.

[18] R. Somoza, P. Conget, F.J. Rubio, Neuropotency of human mesenchymal stem cell cultures: Clonal studies reveal the contribution of cell plasticity and cell contamination, Biol. Blood Marrow Transplant. 14 (5) (2008) 546-555.

[19] Lindstr, et al., Nanoporous titania coating of microwell chips for stem cell culture and analysis, J. Biomech. Sci. Eng. 5 (3) (2010) 272-279.

[20] S. Saberianpour, et al., Tissue engineering strategies for the induction of angiogenesis using biomaterials, J. Biol. Eng. 12 (1) (2018) 36.

[21] H. Hosseinkhani, et al., Engineering three-dimensional collagen-IKVAV matrix to mimic neural microenvironment, ACS Chem. Neurosci. 4 (8) (2013) 1229-1235.

[22] A. Ghodsizadeh, et al., Galactosylated collagen matrix enhanced in vitro maturation of human embryonic stem cell-derived hepatocyte-like cells, Biotechnol. Lett. 36 (5) (2014) 1095-1106.

[23] N. Abbasi, et al., Influence of oriented nanofibrous PCL scaffolds on quantitative gene expression during neural differentiation of mouse embryonic stem cells, J. Biomed. Mater. Res. A 104 (1) (2016) 155-164.

[24] H. Hosseinkhani, et al., Development of 3D in vitro platform technology to engineer mesenchymal stem cells, Int. J. Nanomed. 7 (2012) 3035.

[25] H. Park, et al., Effects of electrical stimulation in C2C12 muscle constructs, J. Tissue Eng. Regenerat. Med. 2 (5) (2008) 279-287.

[26] K. Mareschi, et al., Neural differentiation of human mesenchymal stem cells: evidence for expression of neural markers and eag $\mathrm{K}+$ channel types, Exp. Hematol. 34 (11) (2006) 1563-1572.

[27] H. Dong, W. Shi, T. Bell, Potential of improving tribological performance of UHMWPE by engineering the Ti6Al4V counterfaces, Wear 225 (1999) 146-153.

[28] T. Ishibashi, et al., Astrocytes promote myelination in response to electrical impulses, Neuron 49 (6) (2006) 823-832.

[29] Y. Wu, et al., Mesenchymal stem cells enhance wound healing through differentiation and angiogenesis, Stem Cells 25 (10) (2007) 2648-2659.

[30] Q. Dasgupta, K. Chatterjee, G. Madras, Controlled release of salicylic acid from biodegradable cross-linked polyesters, Mol. Pharm. 12 (9) (2015) 3479-3489.

[31] V.S. Ramachandran, Encyclopedia of the Human Brain, Four-Volume Set, Academic Press, 2002.

[32] J. Sanchez-Ramos, et al., Adult bone marrow stromal cells differentiate into neural cells in vitro, Exp. Neurol. 164 (2) (2000) 247-256.

[33] C. Anacker, et al., Glucocorticoid-related molecular signaling pathways regulating 
hippocampal neurogenesis, Neuropsychopharmacology 38 (5) (2013) 872-883.

[34] B. Mehta, et al., Nitric oxide-mediated modulation of synaptic activity by astrocytic P2Y receptors, J. Gen. Physiol. 132 (3) (2008) 339-349.

[35] B. Mehta, et al., Global Ca2 + signaling drives ribbon-independent synaptic transmission at rod bipolar cell synapses, J. Neurosci. 34 (18) (2014) 6233-6244.

[36] D. Simao, et al., Recapitulation of human neural microenvironment signatures in ipsc-derived NPC 3D differentiation, Stem Cell Reports 11 (2) (2018) 552-564.

[37] J.A. Burdick, G. Vunjak-Novakovic, Engineered microenvironments for controlled stem cell differentiation, Tissue Eng. A 15 (2) (2009) 205-219.

[38] G. Thrivikraman, G. Madras, B. Basu, Electrically driven intracellular and extracellular nanomanipulators evoke neurogenic/cardiomyogenic differentiation in human mesenchymal stem cells, Biomaterials 77 (2016) 26-43.

[39] I.E. Schor, et al., Neuronal cell depolarization induces intragenic chromatin modifications affecting NCAM alternative splicing, Proc. Natl. Acad. Sci. 106 (11) (2009) 4325-4330.

[40] M.C. Pinto, et al., Studying complex system: Calcium oscillations as attractor of cell differentiation, Integr Biol (Camb) 8 (2) (2016) 130-148.

[41] S.S. Rosenberg, N.C. Spitzer, Calcium signaling in neuronal development, Cold Spring Harb Perspect Biol 3 (10) (2011) a004259.

[42] R. McBeath, et al., Cell shape, cytoskeletal tension, and RhoA regulate stem cell lineage commitment, Dev. Cell 6 (4) (2004) 483-495.

[43] L. Song, R.S. Tuan, Transdifferentiation potential of human mesenchymal stem cells derived from bone marrow, FASEB J. 18 (9) (2004) 980-982.

[44] K. Ma, et al., Generation of neural stem cell-like cells from bone marrow-derived human mesenchymal stem cells, Neurol. Res. 33 (10) (2011) 1083-1093.

[45] J. Settleman, Tension precedes commitment—even for a stem cell, Mol. Cell 14 (2) (2004) 148-150.

[46] S. Jain, A. Sharma, B. Basu, Vertical electric field stimulated neural cell functionality on porous amorphous carbon electrodes, Biomaterials 34 (37) (2013) 9252-9263.

[47] G. Thrivikraman, P.K. Mallik, B. Basu, Substrate conductivity dependent mod ulation of cell proliferation and differentiation in vitro, Biomaterials 34 (29) (2013) 7073-7085.

[48] H. Zhao, et al., Specific intensity direct current (DC) electric field improves neural stem cell migration and enhances differentiation towards $\beta$ III-tubulin + neurons, PLoS One 10 (6) (2015) e0129625.

[49] K.E. Hammerick, M.T. Longaker, F.B. Prinz, In vitro effects of direct curren electric fields on adipose-derived stromal cells, Biochem. Biophys. Res. Commun. 397 (1) (2010) 12-17.

[50] M.R. Cho, A review of electrocoupling mechanisms mediating facilitated wound healing, IEEE Trans. Plasma Sci. 30 (4) (2002) 1504-1515.

[51] F. Fröhlich, D.A. McCormick, Endogenous electric fields may guide neocortical network activity, Neuron 67 (1) (2010) 129-143.

[52] C.B. Johansson, et al., Neural stem cells in the adult human brain, Exp. Cell Res. 253 (2) (1999) 733-736.

[53] Y. Levy, et al., Regenerative effect of neural-induced human mesenchymal stromal cells in rat models of Parkinson's disease, Cytotherapy 10 (4) (2008) 340-352.

[54] N. Joyce, et al., Mesenchymal stem cells for the treatment of neurodegenerative disease, Regen. Med. 5 (6) (2010) 933-946.

[55] O. Lindvall, Z. Kokaia, Stem cells in human neurodegenerative disorders-time for clinical translation? J. Clin. Investig. 120 (1) (2010) 29-40.

[56] P. Tropel, et al., Functional neuronal differentiation of bone marrow-derived mesenchymal stem cells, Stem Cells 24 (12) (2006) 2868-2876.

[57] J.E. Snyder, et al., Bioprinting cell-laden matrigel for radioprotection study of liver by pro-drug conversion in a dual-tissue microfluidic chip, Biofabrication 3 (3) (2011) 034112.

[58] G. Thrivikraman, G. Madras, B. Basu, Intermittent electrical stimuli for guidance of human mesenchymal stem cell lineage commitment towards neural-like cells on electroconductive substrates, Biomaterials 35 (24) (2014) 6219-6235.

[59] E.K.F. Yim, S.W. Pang, K.W. Leong, Synthetic nanostructures inducing differentiation of human mesenchymal stem cells into neuronal lineage, Exp. Cell Res. 313 (9) (2007) 1820-1829.

[60] G.J. Her, et al., Control of three-dimensional substrate stiffness to manipulate mesenchymal stem cell fate toward neuronal or glial lineages, Acta Biomater. 9 (2) (2013) 5170-5180.

[61] M. Singh, et al., Synergistic effect of BDNF and FGF2 in efficient generation of functional dopaminergic neurons from human mesenchymal stem cells, Sci. Rep. 7 (1) (2017) 10378 .

[62] L. Danielyan, et al., Survival, neuron-like differentiation and functionality of mesenchymal stem cells in neurotoxic environment: The critical role of erythropoietin, Cell Death Differ. 16 (2009) 1599.

[63] H. Kim, et al., Silicon nanowires promote neuron-like differentiation of mesenchymal stem cells, Biophys. J. 110 (3) (2016) 651a.

[64] R.W. King, T.T. Wu, Electric field induced in cells in the human body when this is exposed to low-frequency electric fields, Phys. Rev. 58 (2) (1998) 2363.

[65] K.R. Robinson, The responses of cells to electrical fields: A review, J. Cell Biol. 101 (6) (1985) 2023-2027.

[66] F. Bezanilla, How membrane proteins sense voltage, Nat. Rev. Mol. Cell Biol. 9 (4) (2008) 323-332.

[67] C.E. Pullar, et al., $\beta 4$ integrin and epidermal growth factor coordinately regulate electric field-mediated directional migration via Rac 1, Mol. Biol. Cell 17 (11) (2006) 4925-4935.

[68] M. Zhao, et al., Membrane lipids, EGF receptors, and intracellular signals colocalize and are polarized in epithelial cells moving directionally in a physiological electric field, FASEB J. 16 (8) (2002) 857-859.

[69] M. Zhao, et al., Electrical stimulation directly induces pre-angiogenic responses in vascular endothelial cells by signaling through VEGF receptors, J. Cell Sci. 117 (3) (2004) 397-405

[70] C.H. Tsai, B.J. Lin, P.H.G. Chao, $\alpha 2 \beta 1$ integrin and RhoA mediates electric field-induced ligament fibroblast migration directionality, J. Orthop. Res. 31 (2) (2013) 322-327.

[71] M. Zhao, et al., Electrical signals control wound healing through phosphatidylinositol-3-OH kinase- $\gamma$ and PTEN, Nature 442 (7101) (2006) 457-460.

[72] S. McLaughlin, M. Poo, The role of electro-osmosis in the electric-field-induced movement of charged macromolecules on the surfaces of cells, Biophys. J. 34 (1) (1981) 85-93.

[73] L.F. Jaffe, Electrophoresis along cell membranes, Nature 265 (5595) (1977) 600-602.

[74] B.-j. Lin, et al., Lipid rafts sense and direct electric field-induced migration, Proc. Natl. Acad. Sci. 114 (32) (2017) 8568-8573.

[75] K.-T. Lim, et al., Pulsed-electromagnetic-field-assisted reduced graphene oxide substrates for multidifferentiation of human mesenchymal stem cells, Adv. Healthc. Mater. 5 (16) (2016) 2069-2079.

[76] H.E. Beggs, et al., NCAM140 interacts with the focal adhesion kinase p125fak and the SRC-related tyrosine kinase p59fyn, J. Biol. Chem. 272 (13) (1997) 8310-8319.

[77] B. Rico, et al., Control of axonal branching and synapse formation by focal adhesion kinase, Nat. Neurosci. 7 (2004) 1059.

[78] R.K. Aaron, et al., Stimulation of growth factor synthesis by electric and electromagnetic fields, Clin. Orthop. Relat. Res. 419 (2004) 30-37.

[79] C.E. Pullar, The Physiology of Bioelectricity in Development, Tissue Regeneration and Cancer, CRC Press, 2016.

[80] F. Li, et al., Superoxide mediates direct current electric field-induced directional migration of glioma cells through the activation of AKT and ERK, PLoS One 8 (4) (2013) e61195.

[81] X. Mu, Y. Li, Conditional TGF-beta 1 treatment increases stem cell-like cell population in myoblasts, J. Cell Mol. Med. 15 (3) (2011) 679-690.

[82] Y. Watanabe, et al., Conversion of myoblasts to physiologically active neurona phenotype, Genes Dev. 18 (8) (2004) 889-900.

[83] D.R. Williams, et al., Synthetic small molecules that induce neurogenesis in skeletal muscle, J. Am. Chem. Soc. 129 (30) (2007) 9258-9259.

[84] D.R. Williams, et al., Fluorescent high-throughput screening of chemical inducers of neuronal differentiation in skeletal muscle cells, Nat. Protoc. 3 (5) (2008) 835-839.

[85] I. Titushkin, M. Cho, Regulation of cell cytoskeleton and membrane mechanics by electric field: Role of linker proteins, Biophys. J. 96 (2) (2009) 717-728.

[86] I. Titushkin, M. Cho, Modulation of cellular mechanics during osteogenic differentiation of human mesenchymal stem cells, Biophys. J. 93 (10) (2007) 3693-3702.

[87] M. Suzuki, C.N. Svendsen, Ex vivo gene therapy using human mesenchymal stem cells to deliver growth factors in the skeletal muscle of a familial ALS rat model, in: F.P. Manfredsson (Ed.), Gene Therapy for Neurological Disorders: Methods and Protocols, Springer New York, New York, NY, 2016, pp. 325-336.

[88] T. Furuta, et al., Mesenchymal stem cell-derived exosomes promote fracture healing in a mouse model, STEM CELLS Transl. Med. 5 (12) (2016) 1620-1630.

[89] S. Dabrowska, et al., Neuroprotective potential and paracrine activity of stromal vs. Culture-expanded hMSC derived from Wharton jelly under Co-cultured with hippocampal organotypic slices, Mol. Neurobiol. 55 (2018) 6021-6036.

[90] C. Sassoli, et al., Bone marrow mesenchymal stromal cells stimulate skeletal myoblast proliferation through the paracrine release of VEGF, PLoS One 7 (7) (2012) e37512.

[91] H. Ryu, et al., Engineering a blood vessel network module for body-on-a-chip applications, J. Lab. Autom. 20 (3) (2015) 296-301.

[92] A. Soto-Gutiérrez, et al., Differentiation of mouse embryonic stem cells to hepatocyte-like cells by co-culture with human liver nonparenchymal cell lines, Nat. Protoc. 2 (2007) 347.

[93] U. Lendahl, L.B. Zimmerman, R.D. McKay, CNS stem cells express a new class of intermediate filament protein, Cell 60 (4) (1990) 585-595.

[94] M.J. Marvin, et al., A rod end deletion in the intermediate filament protein nestin alters its subcellular localization in neuroepithelial cells of transgenic mice, J. Cel Sci. 111 (14) (1998) 1951-1961.

[95] H.-F. Chang, et al., Pulsed DC electric field-induced differentiation of cortical neural precursor cells, PLoS One 11 (6) (2016) e0158133.

[96] K.W. Chang, et al., Fibroblast growth factor-2 up-regulates the expression of nestin through the Ras-Raf-ERK-Sp1 signaling axis in C6 glioma cells, Biochem. Biophys. Res. Commun. 434 (4) (2013) 854-860.

[97] F.B. Sachse, et al., A model of electrical conduction in cardiac tissue including fibroblasts, Ann. Biomed. Eng. 37 (5) (2009) 874-889.

[98] Y. Zhang, et al., Dedifferentiation and proliferation of mammalian cardiomyocytes, PLoS One 5 (9) (2010) e12559.

[99] T. Mantamadiotis, N. Papalexis, S. Dworkin, CREB signalling in neural stem/ progenitor cells: Recent developments and the implications for brain tumour biology, Bioessays 34 (4) (2012) 293-300.

[100] J. Zhou, et al., CREB DNA binding activation by a 50-Hz magnetic field in HL60 cells is dependent on extra-and intracellular Ca $2+$ but not PKA, PKC, ERK, or p38 MAPK, Biochem. Biophys. Res. Biochem. Biophys. Res. Commun. 296 (4) (2002) 1013-1018.

[101] T.A. Brosenitsch, D.M. Katz, Physiological patterns of electrical stimulation can induce neuronal gene expression by activating $\mathrm{N}$-type calcium channels, $\mathrm{J}$. Neurosci. 21 (8) (2001) 2571-2579.

[102] H.M. Blau, C.P. Chiu, C. Webster, Cytoplasmic activation of human nuclear genes in stable heterocaryons, Cell 32 (4) (1983) $1171-1180$. 
[103] T. Tanaka, et al., Alignment of skeletal muscle cells cultured in collagen gel by mechanical and electrical stimulation, Int. J. Tissue Eng. (2014) 52014.

[104] M.H. Thelen, W.S. Simonides, C. van Hardeveld, Electrical stimulation of C2C12 myotubes induces contractions and represses thyroid-hormone-dependent transcription of the fast-type sarcoplasmic-reticulum Ca2 +-ATPase gene, Biochem. J. 321 (Pt 3) (1997) 845-848.

[105] T.T. Schwindt, et al., Effects of FGF-2 and EGF removal on the differentiation of mouse neural precursor cells, An. Acad. Bras. Cienc. 81 (3) (2009) 443-452.

[106] F. Doetsch, et al., EGF converts transit-amplifying neurogenic precursors in the adult brain into multipotent stem cells, Neuron 36 (6) (2002) 1021-1034.

[107] L. Wu, et al., Trophic effects of mesenchymal stem cells in chondrocyte Co-cultures are independent of culture conditions and cell sources, Tissue Eng. A 18 (15-16) (2012) 1542-1551.

[108] Y.S. Takeda, Q. Xu, Neuronal differentiation of human mesenchymal stem cells using exosomes derived from differentiating neuronal cells, PLoS One 10 (8) (2015) e0135111.
[109] H. Haragopal, et al., Stemness enhancement of human neural stem cells following bone marrow MSC coculture, Cell Transplant. 24 (4) (2015) 645-659.

[110] A.F. Adler, et al., Nonviral Direct Conversion of Primary Mouse Embryonic Fibroblasts to Neuronal Cells, vol. 1, Molecular Therapy-Nucleic Acids, 2012

[111] S. Naskar, V. Kumaran, B. Basu, Reprogramming the stem cell behavior by shear stress and electric field stimulation: lab-on-a-chip based biomicrofluidics in regenerative medicine, Regen. Eng. Transl. Med. (2018) 1-29.

[112] M. Wernig, et al., Neurons derived from reprogrammed fibroblasts functionally integrate into the fetal brain and improve symptoms of rats with Parkinson's disease, Proc. Natl. Acad. Sci. 105 (15) (2008) 5856-5861.

[113] R.M. Ferraro, et al., Production of micro-patterned substrates to direct human iPSCs-derived neural stem cells orientation and interaction, Procedia CIRP 65 (2017) 225-230.

[114] J. Chen, et al., Differentiation of mouse induced pluripotent stem cells (iPSCs) into nucleus pulposus-like cells in vitro, PLoS One 8 (9) (2013) e75548. 